\title{
Danke für die Blumen. Kontinuierliches Feedback als Qualitätssicherung im Schreibcoaching
}

\author{
Christina Hollosi-Boiger ${ }^{1}$ \\ Angenommen: 20. November 2020 / Online publiziert: 3. Dezember 2020 \\ (c) Der/die Autor(en) 2020
}

\section{Zusammenfassung}

Schreibcoaching ist eine personenzentrierte, längerfristige Entwicklungs- und Prozessbegleitung mit dem Fokus auf Schreibprozess, schreibender Person und/oder die entstehenden Texte. Aus Sicht des*der Coaches*in ist Schreibcoaching kein einmaliger Vorgang, sondern eine stetige Folge individueller Coachingprozesse. Auf diese sich wiederholenden Prozesse können Ansätze des Qualitätsmanagements übertragen werden, was in der Qualitätsdiskussion im Coaching allgemein bereits geschieht. Das vorgestellte Modell des Qualitätsmanagementsystems für das Schreibcoaching zeigt auf, dass Qualitätsmonitoring in allen Phasen erfolgen und sich konstitutiv auf das Coaching bzw. den Coachingerfolg auswirken kann. Grundlage hierfür bildet ein von dem*der Schreibcoach*in gesteuertes Qualitätskonzept, das Werte, Grundhaltungen und einen operationalisierten Qualitätsbegriff beinhaltet, und in Form eines Leitbilds veröffentlicht werden sollte. Das Fallbeispiel belegt die Umsetzbarkeit des Modells: Qualitätsmessungen aus 22 Schreibcoachings aus dem Bezugsrahmen des beruflichen Schreibens wurden mit der Feedbacktechnik Danke für die Blumen durchgeführt. Diese basiert auf der Blitzlicht-Technik und wurde flächendeckend und kontinuierlich in den Schreibcoachings der Autorin eingesetzt. Die Ergebnisse wurden mittels der inhaltlich strukturierenden, qualitativen Inhaltsanalyse nach Kuckartz rekurrierend auf die Qualitätskriterien im BC-Modell und einer deskriptiv-explorativen statistischen Analyse ausgewertet. Die Ergebnisse zeigen, dass abhängig von den Coachingzielen unterschiedliche Wahrnehmungen zum Schreibcoaching und zur Schreibcoach dominieren, diese sich dennoch über alle Qualitätsdimensionen des Schreibcoachings verteilen. Ein Abgleich mit dem Qualitätskonzept ist daher möglich und kann in einem wiederkehrenden Reflexionsprozess als Entscheidungsgrundlage für das Herbeiführen von Veränderungen herangezogen werden.

Schlüsselwörter Schreibcoaching $\cdot$ Qualitätsmanagementsystem $\cdot$ Evaluation $\cdot$ Feedback $\cdot$ Fallbeispiel

Christina Hollosi-Boiger

post@schreibenmitchribs.at

1 Schreiben mit Chribs, Babenbergerstraße 42/17, 8020 Graz,

Österreich 


\title{
Saying Thank you with Flowers. Continuous Feedback for Quality Assurance in Writing Coaching
}

\begin{abstract}
Coaching for writers is a long-term, person-centered process which focuses on the writing process, writers themselves, and on the texts produced. However, from the coach's point of view, coaching is a steady series of individual coaching sessions. It is a process which can be subjected to a quality management system. In this paper a quality management system tailored for writer coaching is presented. It comprises quality monitoring in all phases of the writer coaching process and impacts the coaching as well as its outcome. The basis is a quality policy managed by the coach, which contains ethics, guiding principles, and an operationalized quality concept, which should be published in form of a quality statement. After the model description a case study proves the feasibility of the model: Evaluation results from 22 writing coachings in the context of professional writing were collected using the feedback technique Thank you for the flowers. This is based on the flash feedback method and was used continuously in the author's writer coaching. The results were evaluated by means of the qualitative content analysis according to Kuckartz, referring to the quality criteria in the BC model and a descriptive-exploratory statistical analysis. The results show that different perceptions of writing coaching and the coach dominate, but that these are still distributed across all quality dimensions. A comparison with the quality policy and its accompanying statement is therefore possible and can be used in a recurring reflection process as basis for the coach's personal improvement.
\end{abstract}

Keywords Writing coaching $\cdot$ Quality management system $\cdot$ Evaluation $\cdot$ Feedback $\cdot$ Case study

\section{Einleitung}

Fröchling (2003) definiert Schreibcoaching als personenzentrierte Beratung Schreibender beim beruflichen, wissenschaftlichen oder literarischen Schreiben. Es handelt sich um eine längerfristige und von mehreren Zusammentreffen geprägte Begleitung und Betreuung von Personen in der Ausübung ihrer Schreibtätigkeit (Klemm 2011). Dies kann neben dem Umfang der Themen (Seidl 2013) als Differenzierungsmerkmal zur Schreibberatung gesehen werden, wobei in der schreibdidaktischen Literatur noch keine ausreichende Begriffsabgrenzung stattgefunden hat (siehe dazu u.a. Brinkschulte et al. (2014), die auch Elemente der Gesprächspsychotherapie, der systemischen Beratung, der kognitiv-behavioristischer Beratung und der Psychoanalyse in der Schreibberatung sehen).

Aus Chirico et al. (2013a) lässt sich ableiten, dass die Themen des Schreibcoachings inputbezogen (Wissen über Schreiben, Schreibprozess, Schreibtechniken), übungsbezogen (Anwenden von Schreibtechniken, Verändern von Schreibverhalten) und personenbezogen (ressourcenaktivierend, reflektierend) sein können. Seidl (2013) führt die Begrifflichkeit Schreibenden Coaching ein, um zu verdeutlichen, dass auch die Person des*der Schreibenden Gegenstand des Coachingprozesses sein kann.

Zentral für die im Schreibcoaching stattfindende Interkation sind die ethischen Grundsätze. So betonen u. a. Grieshammer et al. (2013, S. IX-X), dass der*die Schreibende die Verantwortung für Inhalt und Prozess behält. Meyhöfer (2016, S. 27) sieht darin einen ,schreibprojektbezogenen Handlungsraum“, dessen Ergebnisse in einem interaktiven Konstruktionsprozess entstehen, dem bekannten
Koproduktions-Paradigma (Brandsen et al. 2018). Die Verantwortung für den Text bleibt also bei dem*der Autor*in (Arnold 1999). Kriterien für die Umsetzung der Interaktion fasst Bräuer (2014) als Prinzipien der nicht-direktiven Schreibberatung zusammen: neben dem Prinzip der Hilfe zur Selbsthilfe, sind das die Grundlagen der humanistischen Gesprächstherapie nach Rogers, das angemessene Verhältnis von nicht-direktiven und direktiven Gesprächsanteilen, das Handeln im eigenen Kompetenzbereich und die Einhaltung der organisatorischen Regeln.

Die Autorin selbst definiert Schreibcoaching in Anlehnung an Fröchling (2003) als eine personenzentrierte, längerfristige Entwicklungs- und Prozessbegleitung, die beim Schreiben verschiedenster (beruflicher, wissenschaftlicher, journalistischer, privater bzw. kreativer) Texte unterstützt. Im Fokus des Schreibcoachings können der Schreibprozess, die schreibende Person oder der entstehende Text stehen, wobei diese Themen nicht unabhängig voneinander betrachtet werden können. Dahingehend unterscheidet sich Schreibcoaching auch von anderen Formen des Coachings: Es begleitet den Prozess, nimmt Bezug auf die Person und zugleich auf das Textprodukt, das durch die Coachingbegleitung entstehen soll. Daher kann in Bezug auf Schreibcoaching von einer Mischung aus Fach-, Prozess- und Persönlichkeitsberatung gesprochen werden.

Aus Sicht des*der Coaches*in ist Schreibcoaching kein einmaliger Vorgang, sondern eine stetige Folge individueller Coachingabläufe bestehend aus Vor-, Durchführungsund Nachphase. In diesem Sinne ist die Coachingtätigkeit ein sich wiederholender Prozess. Rascher (2016) kommt zum Schluss, dass die kritische Bewertung der eignen Arbeit „durch die subjektive Einschätzung des Klienten, 
den Abgleich der ursprünglichen Erwartungen mit dem Erreichten oder dem Einholen von Fremdmeinungen erfolgen“ kann. Diesen Ansatz des Qualitätsmanagements unterstreicht auch Anders (2002), der durch die Übertragung von Qualitätselementen auf das Coaching das Anstoßen von Reflexionsprozessen vorschlägt.

Genau an diesem Punkt setzt das nachfolgend vorgestellte Modell eines Qualitätsmanagementsystems für das Schreibcoaching an. Dazu werden zuerst in Abschn. 2 die Phasen des Coachings betrachtet und in Abschn. 3 Qualität im Coaching diskutiert. In Abschn. 4 wird das Modell vorgestellt, welches anhand des Fallbeispiels der Autorin in Abschn. 5 konkretisiert wird.

\section{Phasen des Schreibcoachings}

Im Vergleich der Ablaufmodelle für Schreibcoaching und Schreibberatung zeigt sich, wie sich die Beratungskonstellation und -situation auf die verschiedenen Phasen auswirken. Tab. 1 gibt einen Überblick über drei Modelle des Schreibcoachings (PEACE-Modell von Werder et al. 2001; Phasen des Schreibcoaching-Prozesses, Fröchling 2003; Ablauf des Schreibenden Coaching, Seidl 2013) sowie über zwei Modelle der Schreibberatung (Gestaltung der Schreibberatung, Grieshammer et al. 2013; Ablauf der Beratung, Ulmi et al. 2017). In der Gegenüberstellung wird deutlich, dass sich hinsichtlich des Ablaufs keine klaren Grenzen zwischen Schreibcoaching und Schreibberatung ziehen lassen.

Der Vergleich der Phasen in den Modellen legt zudem offen, dass der Kontext des Schreibens die Interaktion bestimmt und vor allem den Beginn des Coachings beeinflusst: So sind in der Vorphase des Coachings ein Vorgespräch und eine Auftragsklärung nur dann nötig, wenn diese nicht ohnehin aus dem Kontext (z.B. Dozierende Studierende) emergieren. Die Durchführungsphase wird in den angeführten Modellen unterschiedlich ausführlich beschrieben (auf Grund der Zielgruppe der jeweiligen Texte). Allen Modellen gemeinsam ist die Nach- bzw. Abschlussphase, die eine Evaluation bzw. Bewertung des Coachings umfasst: So betonen von Werder et al. (2001) die Wichtigkeit der Evaluation für den*die Schreibcoach*in, um sich weiterzuentwickeln. Als Evaluationsinstrument wird eine dreiphasige Nachbefragung vorgestellt, die einmal direkt in jeder Beratungssitzung, einmal in der letzten Beratungssitzung und schließlich etwa ein halbes Jahr der Beratung stattfindet (vgl. von Werder et al. 2001, S. 155f). Fröchling (2003, S. 165-167) führt Fragen für die Evaluierung an, geht jedoch nicht auf den Umgang mit den erhobenen

Tab. 1 Ablaufmodelle und Phasen in Schreibcoaching und Schreibberatung

\begin{tabular}{|c|c|c|c|c|c|c|}
\hline \multirow[t]{4}{*}{ Phase } & Modell & $\begin{array}{l}\text { PEACE-Modell } \\
\text { für den Prozess des } \\
\text { Schreibcoachings }\end{array}$ & $\begin{array}{l}\text { Phasen des } \\
\text { Schreibcoaching- } \\
\text { Prozesses }\end{array}$ & $\begin{array}{l}\text { Ablauf des Scheibenden } \\
\text { Coachings }\end{array}$ & $\begin{array}{l}\text { Gestaltung der } \\
\text { Schreibberatung }\end{array}$ & $\begin{array}{l}\text { Ablauf der Bera- } \\
\text { tung }\end{array}$ \\
\hline & Quelle & $\begin{array}{l}\text { Von Werder et al. } \\
(2001, \text { S. } 150-165)\end{array}$ & $\begin{array}{l}\text { Fröchling (2003, } \\
\text { S. 151-167) }\end{array}$ & Seidl (2013, S. 14-20) & $\begin{array}{l}\text { Grieshammer } \\
\text { et al. }(2013 \text {, } \\
\text { S. 131-151) }\end{array}$ & $\begin{array}{l}\text { Ulmi et al. (2017, } \\
\text { S. 237-249) }\end{array}$ \\
\hline & $\begin{array}{l}\text { Modell- } \\
\text { bezug }\end{array}$ & $\begin{array}{l}\text { Schreib- und Le- } \\
\text { secoaching, allge- } \\
\text { mein im Kontext des } \\
\text { Schreibens }\end{array}$ & $\begin{array}{l}\text { Schreibcoaching, } \\
\text { allgemein im } \\
\text { Kontext des } \\
\text { Schreibens }\end{array}$ & $\begin{array}{l}\text { Schreibenden Coaching } \\
\text { im akademischen Kon- } \\
\text { text: studentisches Schrei- } \\
\text { ben }\end{array}$ & $\begin{array}{l}\text { Schreibberatung } \\
\text { im akademischen } \\
\text { Kontext: studen- } \\
\text { tisches Schreiben }\end{array}$ & $\begin{array}{l}\text { Schreibberatung } \\
\text { im akademischen } \\
\text { Kontext: studen- } \\
\text { tisches Schreiben }\end{array}$ \\
\hline & $\begin{array}{l}\text { Interak- } \\
\text { tion zwi- } \\
\text { schen }\end{array}$ & $\begin{array}{l}\text { Schreibcoach \& } \\
\text { Klient*in }\end{array}$ & $\begin{array}{l}\text { Schreibcoach \& } \\
\text { Klient*in }\end{array}$ & Coach \& Coachee & $\begin{array}{l}\text { Peer-Tutor*innen } \\
\text { \& Studierende }\end{array}$ & $\begin{array}{l}\text { Dozierende und } \\
\text { Studierende }\end{array}$ \\
\hline \multirow[t]{2}{*}{ Vorphase } & Phase 1 & $\begin{array}{l}\text { P: Problembeschrei- } \\
\text { bung }\end{array}$ & $\begin{array}{l}\text { Vorgespräch/ } \\
\text { Kontaktaufnahme }\end{array}$ & $\begin{array}{l}\text { Vertragsabschluss und } \\
\text { Beziehungsaufbau }\end{array}$ & - & $\begin{array}{l}\text { Beziehung auf- } \\
\text { bauen }\end{array}$ \\
\hline & Phase 2 & $\begin{array}{l}\text { E: Empathie-Ent- } \\
\text { wicklung }\end{array}$ & $\begin{array}{l}\text { Situation analy- } \\
\text { sieren }\end{array}$ & $\begin{array}{l}\text { Anliegen-/ } \\
\text { Auftragsklärung }\end{array}$ & - & $\begin{array}{l}\text { Anliegen konkre- } \\
\text { tisieren }\end{array}$ \\
\hline \multirow[t]{2}{*}{$\begin{array}{l}\text { Durch- } \\
\text { führungs- } \\
\text { phase }\end{array}$} & Phase 3 & $\begin{array}{l}\text { A: Analyse der Pro- } \\
\text { blementwicklung }\end{array}$ & $\begin{array}{l}\text { Ziele und Inhalte } \\
\text { bestimmen }\end{array}$ & $\begin{array}{l}\text { Situationsbeschreibung - } \\
\text { ggf. schreibdidaktische } \\
\text { Diagnostik - Zielfin- } \\
\text { dung - ggfs. Beratung } \\
\text { bzw. schreibdidaktischer } \\
\text { Input }\end{array}$ & $\begin{array}{l}\text { Beratungsgespräch } \\
\text { vorbereiten }\end{array}$ & $\begin{array}{l}\text { Bearbeitungsebene } \\
\text { finden }\end{array}$ \\
\hline & Phase 4 & $\begin{array}{l}\text { C: Coaching des } \\
\text { Problems in Rich- } \\
\text { tung Lösungsange- } \\
\text { bote }\end{array}$ & $\begin{array}{l}\text { Lösungen erar- } \\
\text { beiten }\end{array}$ & Lösungssuche & $\begin{array}{l}\text { Beratungsgespräch } \\
\text { gestalten }\end{array}$ & $\begin{array}{l}\text { Lösungen ent- } \\
\text { wickeln und } \\
\text { Maßnahmen } \\
\text { entscheiden }\end{array}$ \\
\hline Nachphase & Phase 5 & $\begin{array}{l}\text { E: Evaluation der } \\
\text { Schreib- und Lesebe- } \\
\text { ratung }\end{array}$ & $\begin{array}{l}\text { Bewertung und } \\
\text { Abschluss }\end{array}$ & $\begin{array}{l}\text { Planung der ersten Schrit- } \\
\text { te/Abschluss }\end{array}$ & $\begin{array}{l}\text { Beratungsgespräch } \\
\text { nachbereiten }\end{array}$ & Ergebnis sichern \\
\hline
\end{tabular}


Antworten ein. Auch Seidl (2013) beschreibt die Qualitätssicherung nur im Kontext des einzelnen Coachings.

Anhand der Frage der Evaluation wird deutlich, dass qualitätssichernde Bestrebungen im Schreibcoaching vorhanden sind, kontinuierliche Qualitätsmessungen und -sicherungen basierend auf einem Qualitätskonzept jedoch fehlen. Vor diesem Hintergrund stellt sich daher die Frage, wie Qualität etabliert, gemessen, überwacht und gesteigert werden könnte - in allen Phasen des Schreibcoachings. In den oben angeführten Modellen wird zwar die Notwendigkeit der Evaluation thematisiert, vor allem unter dem Aspekt, wann dies im Coaching-Verlauf erfolgen könnte; das Wie und Wozu der Qualitätsmessung wird wenig ausgeführt.

\section{Qualität im (Schreib-)Coaching}

Die Forderung der Qualitätssicherung des Coachings im Allgemeinen wird von verschiedenen Seiten und für verschiedene Qualitätsdimensionen postuliert (u. a. Schermuly und Graßmann 2015). Möller und Kotte (2018) befürworten ein systematisches, diagnostisches Vorgehen, denn dieses könne zur Qualitätssicherung des Coachings beitragen. Diese Erkenntnisse böten auch handlungsleitende Ansätze für praktizierende Coaches und für die Gestaltung von Fortbildungen (Ianiro und Kauffeld 2018).

\subsection{Qualitätsbegriff im Coaching}

Qualität im Coaching lässt sich laut $\mathrm{He} ß$ und Roth (2001) entlang der Qualitätsdimensionen Ergebnis-, Struktur- und Prozessqualität operationalisieren, denen sie über 50 Kriterien zuweisen. Ähnliche Qualitätsdimensionen verwenden Triebel und Heller (2016, S. V-VII.), führen jedoch die Beziehungsqualität als eigene Dimension an. Arnold (1999) schlägt für die Weiterbildung einen viereckigen Qualitätsbegriff vor mit den Dimensionen Legitimations-, Zufriedenheits-, Lern- und Transfererfolg. Laut Anders (2002) hängt der Erfolg von Coaching von der Mitwirkung der Klient*innen ab, weshalb der Qualitätsbegriff im Coaching dialogisch aufzugreifen sei.

Das Konzept der Qualität im Coaching kann demnach umfassend verstanden werden kann; es reduziert sich nicht auf eine Zielerreichung. Rauen (2014) führt aus, dass dieses mehrdimensionale Qualitätsverständnis kontextabhängig betrachtet und mit den Klient*innen individuell abgestimmt werden kann. Ebner (2014) kommt zum Schluss, dass den vielen Aussagen über notwendige Eigenschafen und Fähigkeiten eines*einer Coach*in nur wenig wissenschaftliche Studien zu deren Untermauerung gegenüberstünden. Die „Steuerungsfunktion“ des*der Coaches*in ist laut Webers (2016) geteilt, Anspruch und Verantwortung sollten stattdessen für Coachee-Zentrierung, Coaching-Design und -Umfeld übernommen werden. Die Kompetenz des*der Coaches*in zeige sich daher auch in der Haltung des*der Coach*in.

Die Kompetenz des*der Coaches*in unterstreichen auch Schermuly und Graßmann (2015), die in ihrer Studie zur Wirkung von Coaching feststellen, dass diese Kompetenz in der Drei-Parteien-Konstellation von Coaching (in der Personalentwicklung) die von den Coachees eingebrachten Faktoren (Motivation, Veränderungsbereitschaft und Commitment) und die von der Organisation eingebrachten Faktoren (finanzielle Ressourcen, Unterstützung durch Führung) stütze. Die Kompetenzanforderungen lassen sich z. B. nach Kuchen und Pedrun (2006) in Fach-, Selbst-, Sozial- und Methodenkompetenz einteilen. Erpenbeck und von Rosenstiel (2003) machen deutlich, dass Kompetenz von Qualifikation zu unterscheiden ist und zwischen einem Dispositionsund Performanzeffekt differenziert werden muss. Zurecht erklärt Webers (2016), dass Kompetenzanforderungen weder zeitlos noch kontextfrei seien, sondern in Hinblick auf ihre zeitliche und räumliche Dimension spezifiziert werden müssen. Kompetenz muss aber erst erworben werden, denn, so Berninger-Schäfer (2018), Erwerb und Einsatz der Kompetenz stelle die Voraussetzung für die Einhaltung von Ethikrichtlinien (Grundhaltung und Menschenbild, Fachkompetenz, Vertraulichkeit, Transparenz und Integrität) dar. Die Kompetenz des*der Coaches*in wird deshalb auch in den Qualitätsdimensionen in Abschn. 4 berücksichtigt.

\subsection{Messen der Qualität}

Wenn Coaching als Prozess begriffen wird, können auch prozessorientierte Modelle der Qualitätssicherung angewandt werden. So überträgt Kühl (2008) das in den 1950erJahren entwickelte, auf Trainings ausgelegte Modell von Kirkpatrick (Kirkpatrick 1998) auf das Coaching: die Happiness-Indices ermöglichen eine unmittelbare Reaktion; die Messung von Lernen zeige erweiterte Fähigkeiten, neues Wissen und veränderte Einstellungen; auf der Ebene des Verhaltens werde der Transfer in die organisatorische Praxis und auf der Ebene der Resultate werde ökonomischer Erfolg evaluiert (Kühl 2008). Auch König und Volmer (2002, 2012) differenzieren verschiedene Formen der Evaluation im Coaching und stellen zugleich dar, wann und mit welchem Fokus diese erfolgen können (Ziel-, Prozess-, Input-, Output- und Outcome-Evaluation). Ein weiteres Beispiel zur Steigerung der Prozessqualität ist das erfolgsfaktorenorientierte Prozessmodell von Behrendt (2012; Freiburger Erfolgsfaktoren-Coaching).

Coaching kann auch als eine immaterielle, personenbezogene Dienstleistung verstanden werden (Webers und Zickermann 2019; Schmidt-Lellek 2006). Dieser Betrachtungsweise folgend sind auch die Modelle, Methoden, 
Messinstrumente und Skalen der Qualitätsbestimmung von Dienstleistungen (siehe z.B. Schneider und White 2004; Bueno et al. 2019; oder Ingaldi 2018) für die vorliegende Arbeit zu berücksichtigen.

SERVQUAL (Parasuraman et al. 1985, 1988) ist eines der am häufigsten verwendeten Messinstrumente im Dienstleistungsbereich. Es ist eine 22-teilige, 5-dimensionale Skala, um die kundenseitige Qualitätswahrnehmung der Dienstleistung zu messen. Die fünf Dimensionen sind Rahmenbedingungen (tangibles), Reliabilität (reliability), Offenheit (responsiveness), Vertrauen (assurance) und Empathie (empathy) (Parasuraman et al. 1991). SERVQUAL definiert die Qualität bzw. deren kundenseitige Wahrnehmung auf Basis der Diskrepanz (gaps) zwischen Kundenerwartungen und Durchführung. Das konzeptionelle Lücken-Modell deckt dabei auch Kommunikation und Wahrnehmung vor der eigentlichen Erbringung ab und spricht deshalb auch für das Management der Erwartungshaltungen (Parasuraman et al. 1985). SERVQUAL ist in verschiedene Kontexte und Domänen transferiert und evaluiert worden (Parasuraman et al. 1994; Ladhari 2009) und wird weithin eingesetzt.

Brady und Cronin (2001) stellen ein hierarchisches Modell zur Evaluierung von Dienstleistungsqualität vor (BCModell). Es übernimmt als primäre Dimensionen der Qualitätswahrnehmung jene von Rust und Oliver (1994): die Interaktion bzw. Erbringung der Dienstleistung (interaction quality), Umgebung (physical environment) und Ergebnis (outcome). Diese Dimensionen werden in Anlehnung an Dabholkar et al. (1996) in neun Subdimensionen unterteilt. Die Qualität der Interaktion wird dabei auf Haltung (attitude), Verhalten (behavior) und Expertise (expertise) aufgebrochen. Wie bei SERVQUAL gilt die Skala als valide und reliabel, ist aber nicht ohne Kritik (z.B. Martínez und Martínez 2010a; oder Reimer und Kuehn 2005).

Die vorgestellten Modelle weisen Überschneidungen bei den Dimensionen auf und korroborieren sich damit indirekt gegenseitig; insbesondere die Dimensionen der Interaktion, Expertise und Empathie/Persönlichkeit sind im Fokus. Bueno et al. (2019) kategorisieren die Dimensionen im Zuge einer systematischen Review von 33 Artikeln zur Customer Experience in drei Klassen: Prädisposition, Interaktion und Reaktion. Auch den 30 Studien, die Ladhari (2008) diskutiert, ist gemein, dass sie Qualität als ein mehrdimensionales Konstrukt betrachten. Diese Mehrdimensionalität sowie die hierarchische Struktur bilden die Ausgangsbasis für das in Abschn. 4 vorgestellte Modell. Varnali (2019) setzt diese Differenzierung fort und argumentiert auf Basis der Komplexitätstheorie, dass die Validität der Konstrukte fragwürdig bleiben muss, unter anderem wegen den Prinzipien asymmetrischer Kausalität und der Interdependenz. Eine mögliche Alternative zeigen Martínez und Martínez (2010b) in der phänomenologischen Dekonstruktion der Qualitätswahrnehmung im Kontext einer aus der Ich-Per- spektive von Kund*innen erzählten Consumer Voice auf. Die Autorin hat für ihre Tätigkeit als Schreibcoachin das BC-Modell als Grundlage gewählt (siehe Fallbeispiel in Abschn. 5).

\subsection{Konzepte des Qualitätsmanagements im Coaching}

Der aus Sicht des*der Coaches*in wiederholte Vorgang des Schreibcoachings bietet Potenzial für ständige evidenzbasierte Verbesserung. Es genügt daher nicht, die Qualität des Schreibcoachings punktuell zu erfassen, sondern im Sinne einer Professionalisierung (vgl. Schmidt-Lellek 2006) müssen re- und proaktive Maßnahmen fortwährend umgesetzt werden, was dem klassische Deming-Zyklus (Deming 2000) entspricht. Die PDCA-Abfolge (plan, do, check, act) bildet auch den Kern vieler Standards und Normen, allen voran der ISO 9000-Familie, die als kondensiertes Erfahrungswissen verstanden werden kann. Die ISO 9000:2015 benennt sieben Prinzipien des Qualitätsmanagements (QM), von denen hier auf die Kundenorientierung, Führung und Verbesserung fokussiert werden soll. Es gilt dabei, die meist auf größere Organisationen ausgerichteten QM-Methoden auf das (typischerweise) Einpersonenunternehmen von Coach*innen zu adaptieren. Klute-Wenig und Refflinghaus (2020) gehen der Frage nach, wie das im Kontext von Mikrounternehmen gelingen kann und kommen zum Schluss, dass eine schrittweise Annäherung, welche frühe Erfolge und damit Engagement erzeugen kann, ein wichtiger Erfolgsfaktor sei.

Im Kontext des Coachings identifiziert Steinke (2010) aus drei gängigen QM-Modellen (ISO 9001:2015 2015; EFQM-Modell für Total-Quality-Management; und Kundenorientierte Qualitätstestierung für Beratungsorganisationen (KQB)) jene Elemente, die für ein QM im Coaching genutzt werden können. Steinke (2010) fordert, dass Coach*innen ein eigenes Qualitätsmanagementsystem entwickeln und unterhalten sollten, da dies u.a. als eine kontinuierliche Reflexionsfunktion genützt werden könne.

Ein solches Qualitätsmanagementsystem beinhaltet laut ISO-Norm unter dem Aspekt der Führung das „Kommunizieren der organisationseigenen Mission, Vision, Strategie, Politiken und Prozesse“ durch „Werte, Fairness und Leitbilder ethischen Verhaltens“ (ISO 9000:2015 2015). Kommunikation beginnt dabei schon im Vorfeld, beim ersten Informationskontakt (Wastian und Poetschki 2016). Eine transparente Informationsgestaltung - bspw. in Form eines Coachingkonzepts - zählt daher zu den Kernkompetenzen des*der Coaches*in (Kuchen und Pedrun 2006). Im Kontext der ISO 9001:2015 kann hier von einer Qualitätspolitik gesprochen werden. Für ein ausgearbeitetes und offen gelegtes Coachingkonzept plädiert auch Schreyögg (2000), da es Transparenz für den Coaching-Suchenden bietet. Ein sol- 
ches Coachingkonzept solle vor allem die Werte des*der Coaches*in offenlegen, damit eine Kongruenz zwischen den Werten der Coaching-Suchenden und der Anbietenden ermöglicht werde (Hanke und Boehnke 2018). Lippmann (2015) führt aus, dass die Coaching-Qualifizierung mit umfassenden Inhalten und Themengebieten auf die Entwicklung eines Coachingkonzepts abzielen solle, was zu einer Professionalisierung im Coaching führen würde.

\section{Modell des Qualitätsmanagements für das Schreibcoaching}

Wie in Abschn. 2 dargelegt wird in den Ablaufmodellen zum Schreibcoaching bzw. in der auf coachende Haltung fokussierten Schreibberatung eine Evaluation angedacht (vgl. u. a. dazu von Werder et al. 2001; Fröchling 2003; Seidl 2013; Grieshammer et al. 2013; Ulmi et al. 2017), wobei diese in der Abschlussphase des Schreibcoachings zu verorten ist und sich vorwiegend auf die Ergebnisqualität des Coachings bezieht (vgl. dazu auch Klemm 2011). Während von Werder et al. (2001, S. 155f.) von einer Qualitätsüberprüfung und einem Ansporn zur Entwicklung sprechen, führen nur Grieshammer et al. (2013, S. 150) aus, dass die Dokumentation der Durchführung zur „Reflexion anregen und den Austausch mit anderen Schreibberatenden ermöglichen" soll. Eine kontinuierlich angewandte und theoretisch fundierte Vorgehensweise des Qualitätsmanagements im Schreibcoaching mit Qualitätskriterien als Basis der Qualitätsüberprüfung sowie Methoden der Evaluierung werden derzeit in der Literatur noch wenig beachtet ${ }^{1}$.

\subsection{Qualitätsdimensionen und Evaluationsformen}

Auch der Qualitätsbegriff im Schreibcoaching bedarf einer differenzierteren Segmentierung als in der Literatur bisher vorgestellt. Tab. 2 stellt eine solche Strukturierung vor. Die aus der Coaching-Literatur bekannten Dimensionen der Struktur-, Beziehungs-, Prozess- und Ergebnisqualität (Spalte 1 der Tabelle; vgl. Heß und Roth 2001; sowie Triebel und Heller 2016, S. V-VII) bzw. den drei Dimensionen des BC-Modells (Brady und Cronin 2001) in Spalte 2 werden in Spalte 3 weiter differenziert bzw. präzisiert, indem vor allem die Beziehungsqualität in die Dimensionen Interaktionsqualität (vgl. Bueno et al. 2019), personale Qualität (vgl. Ebner 2014; sowie Webers 2016) und Coach-Kompetenz (vgl. Schermuly und Graßmann 2015) aufgeschlüsselt wird. Damit wird sichtbar, dass einerseits die Person des*der Coach*in wesentlichen Einfluss auf die Konstellation im Coaching hat und dass anderer-

${ }^{1}$ Einzig Fröchling (2003) spricht von Qualitätskriterien im Schreibcoaching, die auf mehreren Qualitätsdimensionen zu verorten sind. seits im Schreibcoaching (in Abgrenzung zu generischem Coaching) eine fachliche bzw. methodische Kompetenz nötig ist und auch Einsatz findet (vgl. Abschn. 1 bzw. Subdimension expertise im BC-Modell). In der Strukturqualität wurde zudem die Umgebung als Merkmal explizit angeführt, da Schreibcoachings auch vor Ort bei Coachees stattfinden können.

Die Qualitätsdimensionen im Schreibcoaching haben mehrere Adressat*innen (Coach*in, Coachee, Auftraggeber*in) und können auf unterschiedliche Art gemessen werden. Beispielsweise können mündliche oder schriftliche Befragungen in einem Interview-, Fragebogen- oder Fokusgruppen-Setting durchgeführt werden. In Anlehnung an die Evaluationsformen im Coaching nach König und Volmer (2002, 2012) und das PEACE-Modell von Werder et al. (2001) werden unter Berücksichtigung der Ausführungen von Fröchling (2003) die Evaluationsformen in Tab. 3 zusammengefasst. Diese Übersicht dient als Orientierung, um den modus operandi der Qualitätsmessung zu definieren.

\subsection{Qualitätskonzept und Qualitätsmanagementsystem}

Ausführungen zur Qualität im Coaching weisen deutlich darauf hin, dass eine Offenlegung der Qualitätsprinzipien sinnvoll und notwendig ist (u.a. Schreyögg 2000; Rascher

Tab. 2 Qualitätsdimensionen im Schreibcoaching

\begin{tabular}{|c|c|c|}
\hline $\begin{array}{l}\text { Qualitäts- } \\
\text { dimensionen } \\
\text { im Coa- } \\
\text { ching }\end{array}$ & $\begin{array}{l}\text { Qualitätsdimension } \\
\text { im BC-Modell }\end{array}$ & $\begin{array}{l}\text { Qualitätsdimensionen im } \\
\text { Schreibcoaching }\end{array}$ \\
\hline $\begin{array}{l}\text { Struktur- } \\
\text { qualität }\end{array}$ & $\begin{array}{l}\text { Physical environment } \\
\text { quality: } \\
\text { ambient conditions, } \\
\text { design, social factors }\end{array}$ & $\begin{array}{l}\text { Strukturqualität: } \\
\text { Coaching-Setting, -Umstän- } \\
\text { de und -Umgebung }\end{array}$ \\
\hline \multirow[t]{3}{*}{$\begin{array}{l}\text { Beziehungs- } \\
\text { qualität }\end{array}$} & $\begin{array}{l}\text { Interaction Quality: } \\
\text { attitude, behavior, } \\
\text { expertise }\end{array}$ & $\begin{array}{l}\text { Interaktionsqualität: } \\
\text { Einstellung, Grundhal- } \\
\text { tung, Verhalten des*der } \\
\text { Coach*in, Beziehungsauf- } \\
\text { bau }\end{array}$ \\
\hline & & $\begin{array}{l}\text { Personale Qualitäten: } \\
\text { Persönlichkeit, Eigenschaf- } \\
\text { ten, Auftreten, Authentizität }\end{array}$ \\
\hline & & $\begin{array}{l}\text { Coach-Kompetenz: } \\
\text { Methodenkompetenz, kultu- } \\
\text { relle Kompetenz, Feldkom- } \\
\text { petenz, } \\
\text { Fachkompetenz }\end{array}$ \\
\hline $\begin{array}{l}\text { Prozess- } \\
\text { qualität }\end{array}$ & - & $\begin{array}{l}\text { Prozessqualität: } \\
\text { Coaching-Design, Coa- } \\
\text { ching-Technik, Verlauf des } \\
\text { Coachingprozesses }\end{array}$ \\
\hline $\begin{array}{l}\text { Ergebnis- } \\
\text { qualität }\end{array}$ & $\begin{array}{l}\text { Outcome quality: } \\
\text { social factors, wai- } \\
\text { ting time, tangibles, } \\
\text { valence }\end{array}$ & $\begin{array}{l}\text { Ergebnisqualität: } \\
\text { Zielerreichung, Zufrieden- } \\
\text { heit, Transfer, Textprodukt }\end{array}$ \\
\hline
\end{tabular}




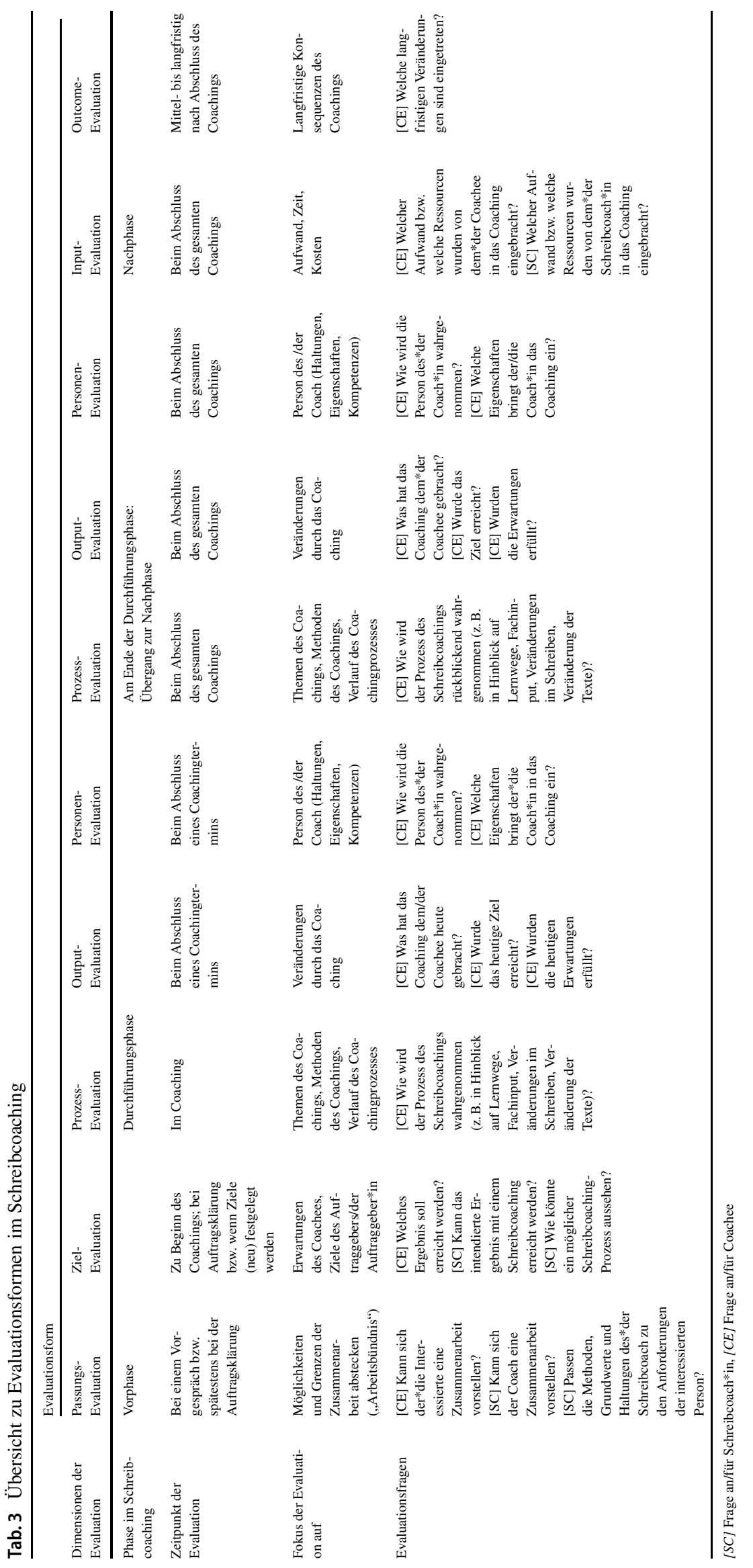




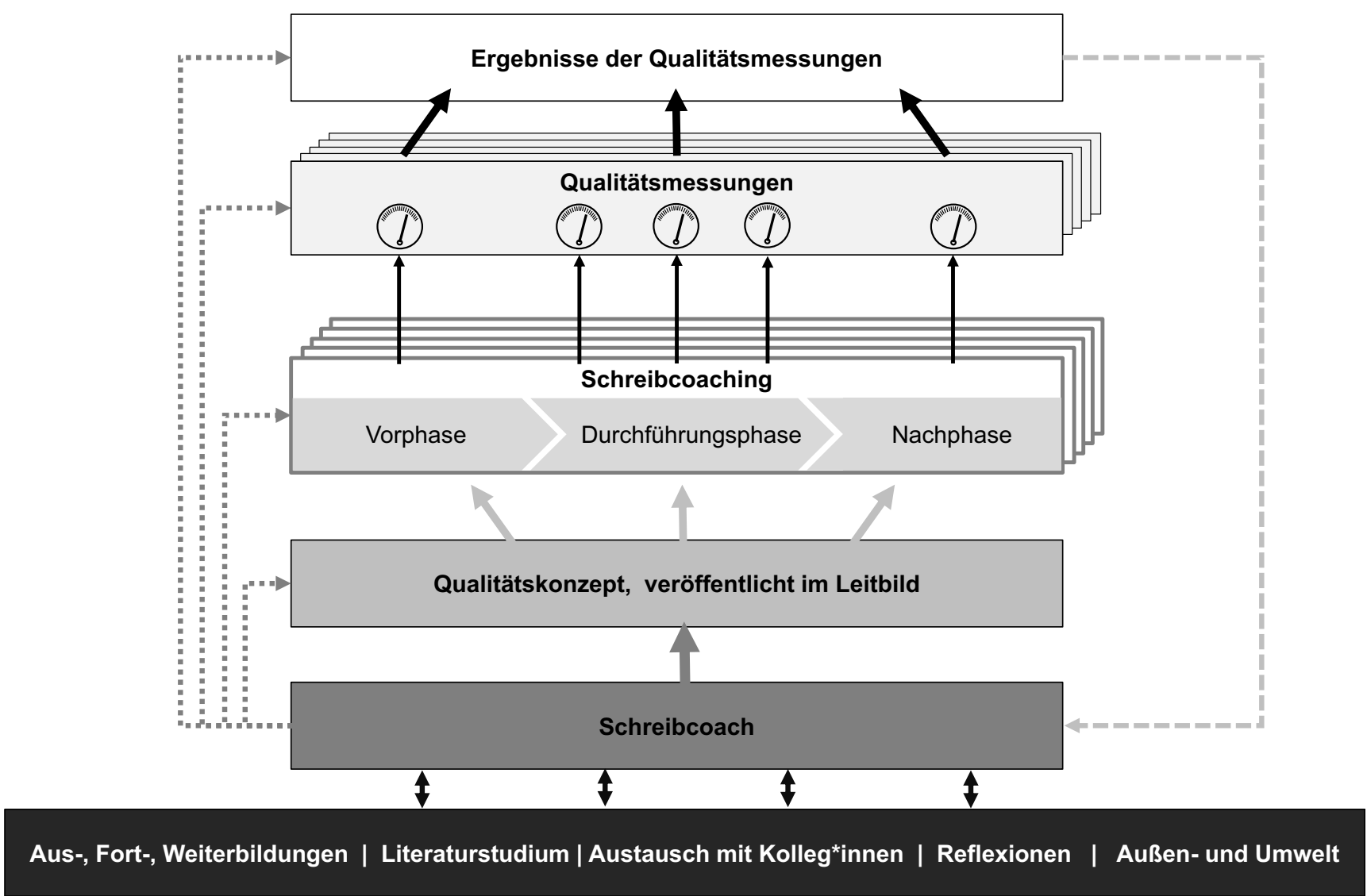

Abb. 1 Qualitätsmanagementsystem für das Schreibcoaching

2016; Hanke und Boehnke 2018; Rojon et al. 2020). Dies erfolgt klassischerweise im Coachingkonzept, welches in und durch eine entsprechende Qualifizierung etabliert werden solle (Lippmann 2015). In der Qualitätsdiskussion im Bereich des Coachings wird nicht nur eine Evaluation gefordert (u.a. Heller und Gallenmüller 2016; Klonek et al. 2016), sondern auch gezeigt, dass Evaluation im gesamten Coaching-Prozess erfolgen kann und dort schon konstitutiv auf das Coaching bzw. den Coachingerfolg wirken kann (vgl. Leedham 2005; König und Volmer 2002, 2012; Schmidtmayer 2016).

Das in Abb. 1 vorgestellte Qualitätsmanagementsystem für das Schreibcoaching zeigt auf, wie und in welchen Dimensionen Qualität im Schreibcoaching präsent ist, zu welchem Zeitpunkt Qualität gemessen werden kann und was mit den Ergebnissen dieser Evaluationen geschehen kann.

Von dem*der Schreibcoach*in wird ausgehend von der Coachingqualifizierung ein Qualitätskonzept entworfen und verschriftlicht, welches die Werte und Haltungen beinhaltet und definiert, was als Qualität verstanden und wie diese gemessen wird. Als Ausgangsbasis schlage ich vor, die Foundation Factors von Leedham (2005) zu verwenden, die er in Adaption der Smart Performance Pyramid von Cross und Lynch (1988) definiert: Effektives Coaching beruhe auf
Kompetenz und den persönlichen Eigenschaften des*der Coach*in, auf dem Coachingprozess und der Coachingumgebung. Hier sind auch die Werte, die Grundhaltungen, das Menschenbild sowie die Grundprinzipien der Vertraulichkeit, Transparenz und Integrität (vgl. Berninger-Schäfer 2018) zu verorten. Ausgehend von diesen immateriellen Werten, können die Qualitätsdimensionen (aus Abschn. 4.1) hinsichtlich der Evaluationsmöglichkeiten operationalisiert und messbar gemacht werden.

Schreyögg (2000) schlägt für die Veröffentlichung der Werte und Haltungen ein Coachingkonzept vor. Für das Schreibcoaching rege ich die Verwendung eines Leitbildes an, da es die zusätzlichen Informationen der eigenen Stärkefelder (und somit eine Positionierung) sowie den Zweck der angebotenen Dienstleitungen beinhalten kann. Dies erleichtert dem*der Schreibcoach*in, das Leitbild auf ev. weitere angeboten Dienstleistungen mit ähnlichen Grundhaltungen und Werten (wie etwa Schreibberatung oder Schreibtraining) auszuweiten und diese zugleich $\mathrm{zu}$ differenzieren. Das Leitbild stellt eine Reduktion des Qualitätskonzeptes dar und soll - entsprechend der Forderungen der ISO 9001:2015 und Schreyögg (2000) - veröffentlicht werden. Koubek (2015) zeigt für die Bekanntmachung verschiedene 
Wege auf, bspw. auf einer Website, in persönlichen Gesprächen etc.

Das Qualitätskonzept wirkt auf jede Phase und kann folglich auch in jeder Phase des Schreibcoachings gemessen werden. In der Vorphase können sich Interessierte über das veröffentlichte Leitbild im Sinne einer kundenorientierten Transparenz informieren, was zu einer besseren Passung zwischen Schreibcoach*in und Coachee führt (siehe dazu Kuchen und Pedrun 2006; Schreyögg 2000; Hanke und Boehnke 2018). Diese Evaluationsform führe ich als Passungs-Evaluation ein. In Anlehnung an König und Volmer $(2002$, 2012) erfolgt zu Beginn des Coachings auch eine Ziel-Evaluation, die abklären muss, ob das intendierte Ergebnis überhaupt durch das Schreibcoaching erreicht werden kann.

Auch in der Durchführungsphase ist das Qualitätskonzept ständig präsent, da es die Basis für die eingebrachte Haltung ins Coaching und dessen Verlauf ist, was als Prozessqualität gemessen werden kann. Während des Coachings kann auch die Ergebnisqualität bzw. Zielerreichung (also eine Output-Evaluation) geprüft werden, die von Werder et al. (2001) als erste Nachbefragung im PEACE-Modell angegeben wird. Durch die Möglichkeit der direkten und unmittelbaren Evaluation im jeweiligen Coachingtermin, können Korrekturen rasch vorgenommen und qualitätssichernde Maßnahmen gleich realisiert werden. Während der Durchführung des Coachings kann auch eine personenzentrierte Evaluation in Hinblick auf die Eigenschaften des*der Coaches*in stattfinden. Wie bereits dargelegt, wird mehrfach auf die zentrale Bedeutung der Person des*der Coaches*in und auf der die Interaktion eines Coachings prägenden Haltungen und Eigenschaften hingewiesen. Diese Evaluationsform führe ich als Personen-Evaluation ein, die sowohl in der Durchführungsphase als auch am Ende der Durchführungsphase stattfinden kann.

Am Ende der Durchführungsphase, also beim Abschluss des Schreibcoachings, kann ein weiteres Mal nach der Ergebnisqualität gefragt werden (Output-Evaluation im Sinne der zweite Nachbefragung des PEACE-Modells: „Was nimmt der*die Coachee mit?"), genauso wie nach der Prozessqualität (Prozess-Evaluation: Wie wird der Coachingprozess rückblickend wahrgenommen?). Diese das Schreibcoaching abschließende Qualitätskontrolle gibt Aufschluss über die Effektivität des Coachings.

In der Nachphase zum Schreibcoaching kann schließlich nach den eingebrachten Inputs und Ressourcen gefragt werden (Input-Evaluation) sowie nach den Auswirkungen des Coachings (Outcome-Evaluation), d.h. dem mittel- bis langfristigen Konsequenzen des Coachings (was der dritten Nachbefragung im PEACE-Modell entspricht).

Die gesammelten Ergebnisse der Evaluationen können in die Arbeit des*der Schreibcoaches*in einfließen und die Qualität des aktuellen bzw. aller folgenden Schreibcoa- chings prägen. Während in der Coachingliteratur von der Weiterentwicklung des*der Coaches*in gesprochen wird und diese auch eingefordert wird (Schmidt-Lellek 2006), zeigen Peters und Girgensohn (2012) und Chirico et al. (2013b), was auch schon von Werder et al. (2001) angedeutet haben: Die Reflexion der Evaluationsergebnisse führt zu Veränderungen. Peters und Girgensohn (2012) beschreiben, wie sie diese durch Reflexion wieder in die Tätigkeit der Schreibberatung einfließen lassen, unter anderem im persönlichen Kontakt, bei der Umgestaltung der Flyer etc. Auch Chirico et al. (2013b) führen Schlussfolgerungen aus Evaluationen wieder in die Aus- oder Weiterbildung von Schreibberater*innen rück.

Für das Schreibcoaching plädiere ich für eine beständige Evaluation und eine einheitliche, systematische Sammlung, Auswertung und Analyse der Messergebnisse, um die eigene Qualität evidenzbasiert und kontinuierlich zu beobachten. Anschließend an dieses Qualitätsmonitoring können die Ergebnisse reflektiert und diskutiert, als Impuls in eine Supervision oder Intervision eingebracht und ggfs. auf einer weiteren Evaluationsebene (z. B. Fokusgruppe mit einzelnen Coachees) hinterfragt werden. Im Qualitätsmanagement würde dieses Innehalten, dieses Sich-der-Qualität-desCoachingsprozesses-Bewusst-Werdens einer Managementbewertung (einem Management Review) entsprechen und $\mathrm{zu}$ einer Selbstbewertung führen, die auch eine Betrachtung des Reifegrads des Qualitätsmanagementsystems in Anlehnung an ISO 9004:2018 (2018) bzw. eines EFQM-Modelles (2013) beinhalten würde.

Das vorliegende Modell sieht den*die Schreibcoach*in als Dreh- und Angelpunkt, der*die die Qualität der Dienstleistung „Schreibcoaching“ steuern kann, nämlich in jenen Bereichen, für die innerhalb einer Koproduktion mit dem Coachee Verantwortung übernommen werden kann (siehe Abschn. 1). Wie bereits ausgeführt, mag die Aufgabe ein Qualitätsmanagement als Einzelperson zu betreiben, als zu große Herausforderung erscheinen. Im Folgenden soll daher anhand des Fallbeispiels der Autorin gezeigt werden, wie ein mit einfachen Methoden erhobenes Feedback dazu beitragen kann, „Werte und Kultur zu prägen und weiterzuentwickeln" (Koubek 2015) und so der Forderung Steinkes (2010, S. 436) nachzukommen, dass jeder*jede Coach*in „ein eigenes professionelles Qualitätsmanagementsystem entwickeln und unterhalten“" sollte.

\section{Fallbeispiel anhand der Schreibcoachings der Autorin}

Das vorliegende Fallbeispiel präsentiert Methode und Ergebnisse der Qualitätsmessung der Autorin im Bereich des Schreibcoachings für berufliches Schreiben. Zentraler Aspekt des Qualitätsmanagementsystems der Autorin 
ist die Qualitätspolitik (vgl. ISO 9001:2015 2015). In Abschn. 5.1 werden daher zuerst die Qualitätspolitik und -dimensionen dargelegt und in Abschn. 5.2 die Prinzipien der Qualitätsmessung der Autorin, bevor die Evaluationstechnik „Danke für die Blumen“ in Abschn. 5.3 dargestellt wird. Die Eckdaten des so erfassten Datensatzes werden nachfolgend in Abschn. 5.4 beschrieben und die Daten der Rückmeldungen inhaltlich (Abschn. 5.5) und statistisch (Abschn. 5.6) ausgewertet. Abschn. 5.7 zeigt abschließend, wie die so gewonnen Erkenntnisse wieder ins Qualitätsmanagement rückgeführt werden. Die Aufteilung folgt damit den Funktionen der Evaluierung nach Kromrey (2001, S. 110).

\subsection{Qualitätsdimensionen und Qualitätspolitik}

Ausgangspunkt für die Qualitätspolitik der Autorin bildeten die Qualitätsdimensionen im Schreibcoaching (siehe Abschn. 4.1). Anhand dieser hat die Autorin die Ansprüche an die von ihr durchgeführten Schreibcoachings formuliert (siehe Tab. 4). Auf Basis dieser Politik wurde in Folge das Qualitätskonzept in Form eines Leitbildes verankert. Es beinhaltet Mission, Vision und Grundwerte, die mit den ethischen Grundsätzen kongruieren (vgl. Wagner und Patzak 2015; bzw. Kaplan und Norton 2004). Das publizierte Leitbild $^{2}$ dient bereits in der Orientierungsphase der*dem potenziellen Coachee bei der Auswahl bzw. dem Management der Erwartungshaltung (vgl. Parasuraman et al. 1985).

Hervorzuheben ist, dass es sich bei der Erstellung eines Qualitätskonzeptes um einen rekursiven, mehrdimensionalen, analytischen Prozess handelt: Neben der Kompetenz-(weiter-)entwicklung erfolgte eine Bewusstwerdung der Kernkompetenzen und Fokussierung im Tätigkeitsfeld (in Anlehnung an das wertstromorientierte Prozessmanagement, vgl. Wagner und Patzak 2015, S. 231-244). Parallel dazu wurde für das Qualitätskonzept auch eine markenstrategische Positionierung durchgeführt, die an immateriellen Vermögenswerten im Sinne einer Strategy Map (vgl. Kaplan und Norton 2004) orientiert ist. Das Qualitätskonzept der Autorin entstand also durch den Erwerb von Kompetenzen - was Berninger-Schäfer (2018) als Voraussetzung für die Einhaltung von Ethikrichtlinien darstellt -, die Reflexion derselben - also dem, was Erpenbeck und von Rosenstiel (2003) als Differenz zwischen Dispositions- und Performanzeffekt bezeichnen - sowie durch eine Analyse der Geschäftsprozesse - entlang der Qualitätsdimensionen im Schreibcoaching.

Im Sinne des in Abschn. 4 vorgestellten Qualitätsmanagementsystems für das Schreibcoaching kommt der Qualitätsmessung ein hoher Stellenwert zu. Rückmeldungen der Coachees sowie alle anderen, zusätzlichen Messergebnisse

${ }^{2}$ Das Leitbild ist unter anderem auf der Website der Autorin publiziert.
Tab.4 Qualitätsdimensionen und Qualitätspolitik der Autorin

\begin{tabular}{|c|c|}
\hline $\begin{array}{l}\text { Qualitätsdimensionen } \\
\text { des Schreibcoaching }\end{array}$ & $\begin{array}{l}\text { Ansprüche der Autorin an Coaching } \\
\text { (Qualitätspolitik der Autorin) }\end{array}$ \\
\hline $\begin{array}{l}\text { Strukturqualität } \\
\text { Coaching-Setting, } \\
\text {-Umstände } \\
\text { und -Umgebung }\end{array}$ & $\begin{array}{l}\text { Setting: durchdacht \& konzipiert; } \\
\text { Flexibles Eingehen auf Coachee, Situati- } \\
\text { on, Setting } \\
\text { Arbeitsumgebung schaffen, Schreibinfra- } \\
\text { struktur etablieren }\end{array}$ \\
\hline $\begin{array}{l}\text { Interaktionsqualität } \\
\text { Einstellung, Grund- } \\
\text { haltung und Verhalten } \\
\text { des*der Coach*in, } \\
\text { Beziehungsaufbau }\end{array}$ & $\begin{array}{l}\text { Hilfe zur Selbsthilfe } \\
\text { Personenzentrierte Beratung nach Rogers } \\
\text { Gegenseitige Akzeptanz } \\
\text { Wertschätzung von Schreibenden und } \\
\text { Texten } \\
\text { Vertrauensprinzip } \\
\text { Kompetenzbereiche beachten, im eigenen } \\
\text { Kompetenzbereich bleiben }\end{array}$ \\
\hline $\begin{array}{l}\text { Personale Qualitäten } \\
\text { Persönlichkeit, Eigen- } \\
\text { schaften, Auftreten, } \\
\text { Authentizität }\end{array}$ & $\begin{array}{l}\text { Schreiblust spürbar/erlebbar machen } \\
\text { Mit Humor geht vieles leichter! } \\
\text { Klarheit \& Fokussierung } \\
\text { Empathie } \\
\text { Kongruenz und Echtheit }\end{array}$ \\
\hline $\begin{array}{l}\text { Coach-Kompetenz } \\
\text { Methodenkompetenz, } \\
\text { kulturelle Kompetenz, } \\
\text { Feldkompetenz, Fach- } \\
\text { kompetenz/fachlicher } \\
\text { Input }\end{array}$ & $\begin{array}{l}\text { Hoher Stellenwert von Aus- \& Weiterbil- } \\
\text { dung } \\
\text { Prozessorientierter Aufbau des Coachings } \\
\text { Exemplarisches Anwenden von Strategien, } \\
\text { Methoden und Techniken im Coaching } \\
\text { Stellenwert der eigenen Schreibkompetenz } \\
\text { Feldkompetenz in verschiedenen Domä- } \\
\text { nen des Schreibens } \\
\text { Fachliche Expertise im Schreiben }\end{array}$ \\
\hline $\begin{array}{l}\text { Prozessqualität } \\
\text { Coaching-Design }\end{array}$ & $\begin{array}{l}\text { Bei Diagnostik, Konzeption, Vorbereitung } \\
\text { und Nachbereitung Kund*innen-Perspek- } \\
\text { tive einnehmen } \\
\text { Klare Struktur im Schreibcoaching, Füh- } \\
\text { rung durch Coachin } \\
\text { Transparenz in Methodik, Struktur, Ablauf } \\
\text { durch Qualitätsmanagement }\end{array}$ \\
\hline $\begin{array}{l}\text { Ergebnisqualität } \\
\text { Zielerreichung, Zu- } \\
\text { friedenheit, Wiederbe- } \\
\text { auftragung, Transfer, } \\
\text { Textprodukt }\end{array}$ & $\begin{array}{l}\text { Wirkung in Ziele miteinbeziehen } \\
\text { Messbar und sichtbar machen (abhängig } \\
\text { von Auftragsart) } \\
\text { Folgeangebote für Validieren des Trans- } \\
\text { fers }\end{array}$ \\
\hline
\end{tabular}

fließen im Sinne eines PDCA-Zyklus (Deming 2000) wieder in die schreibdidaktische Arbeit der Autorin rück. Dazu werden die ausgewerteten Daten in periodisch wiederkehrenden Momenten mit Hilfe einer individuell gestalteten Form der Balanced Scorecard (siehe dazu Koubek 2015), sowie Ausführungen zur Coaching Scorecard von Leedham (2005) analysiert und mit iterativen Reflexionsprozessen gestützt. So finden nicht nur Evaluationsergebnisse, sondern auch die Eigenwahrnehmung der Autorin und von Auftraggeber*innen durchgeführte Evaluationsergebnisse Eingang in die Analyse.

Schließlich werden die Qualitätskriterien mit den Ergebnissen der Qualitätsmessungen abgeglichen. Die auf diese Weise ermittelten Informationen werden in Form einer Managementbewertung mit Ergebnissen früherer Qualitätsmessungen sowie weiterer Key Performance Indicators ver- 
glichen und bilden so die Entscheidungsgrundlage für das Herbeiführen von Veränderungen, etwa durch Fortbildungen, durch vertiefende Literaturstudien, durch den Austausch mit Kolleg*innen, durch Supervision o. Ä. Dieses Vorgehen intendiert die kontinuierliche Verbesserung des Schreibcoachings, der Adaptierung und Präzisierung des Qualitätskonzepts sowie der Weiterentwicklung der Coachin selbst. U. a. wird dieser Prozess durch das Führen eines schreibdidaktischen Journals reflektierend begleitet.

\subsection{Design, Ziele und Setting der Qualitätsmessung}

Zur Qualitätsmessung setzt die Autorin verschiedene Mess-, Analyse- und Bewertungsinstrumente ein. Zentral dabei ist, dass diese Beobachtungs- und Bewertungsmomente nicht singulär stattfinden, sondern verteilt über das gesamte Coaching, in unterschiedlichen Formen und Ebenen (vgl. dazu auch Tab. 3 in Abschn. 4). Qualität ist damit integrativer Bestandteil und Kernfaktor für das Professionalitätsverständnis der Autorin (vgl. Schmidt-Lellek 2006). Die Operationalisierung der Qualitätsdimensionen für die Messung gestaltet sich dabei unterschiedlich. So können bspw. Ergebnis- und Wirkqualität bei Coachings zu Textund Schreibkompetenz einerseits an fertigen Textprodukten gemessen werden. Andererseits kann bei Coachings zu Klarheit und Identifikation von Schreibproblemen nur die*der Coachee selbst die erreichte Qualität beurteilen.

Für die begleitende, formative Evaluierung (vgl. z.B. Bortz und Döring 2006, S. 110) hat die Autorin (unabhängig von Methode und Form) folgende Rahmenbedingungen für ihre Qualitätsmessung definiert:

- Passung zu Dimensionen der Qualitätspolitik: Messungen müssen auf die Dimensionen des Schreibcoachings rekurrieren, da sie sonst nicht im Qualitätsmanagementsystem behandelt werden können.

- Umsetzbarkeit: Als Einpersonenunternehmen kann die Autorin nur begrenzte Ressourcen für die Evaluation aufbringen. Die Erstellung, Durchführung und Auswertung muss daher entsprechend effizient möglich sein und quick wins erlauben (vgl. Klute-Wenig und Refflinghaus 2020).

- Verhältnismäßigkeit: Die Dauer der Evaluierung darf im Verhältnis zur Dauer einer Coaching-Sitzung keine substanzielle Zeit beanspruchen. ${ }^{3}$

- Hoher Rücklauf: Die Methode soll zu Feedback und Evaluation animieren, nicht ob ihrer Form von der Teilnahme abschrecken.

\footnotetext{
${ }_{3}^{3}$ Beispielsweise wären 15 min Evaluation bei einem 60 -minütigen Coaching unangebracht.
}

- Offen: Die Evaluation soll Coachees ermöglichen, Feedback zu Punkten zu geben, die noch nicht oder in zu geringem Umfang vom Qualitätsmanagement erfasst sind, um das QM selbst iterativ verbessern zu können.

\subsection{Die Evaluationstechnik „Danke für die Blumen“}

Mit den in Abschn. 5.2 genannten Rahmenbedingungen, die Evaluation im Schreibcoaching sowohl im Prozess als auch in der Abschlussphase des Schreibcoachings mit einfachen Mitteln und flächendeckend durchzuführen, wurde basierend auf der Blitzlicht-Technik die Evaluationstechnik Danke für die Blumen als zweigliedriges Feedbackverfahren entwickelt.

Originäre Quellen zur Blitzlicht-Technik sind nicht auszumachen, jedoch wird bereits von Cohn (1975) eine Blitzaufnahme mit der Frage ,Was haben Sie eben in diesem Augenblick erlebt - oder im Bewusstsein gehabt?" (Cohn 1975, S. 117) erwähnt. Wesensmerkmal des Blitzlichts ist eine kurze Antwort, eine der Konstellation inhärente Umfangsbeschränkung, die sich dazu eignet, flüchtige Wahrnehmungen zu ermitteln. Da ein solches Blitzlicht rasch durchzuführen ist, kann es am Ende jeder Coachingsitzung abgefragt werden, was sich positiv auf die Akzep$\operatorname{tanz}$ auswirkt. Um die Evaluation im Sinne des Qualitätskonzepts der Autorin nachvollziehbar und auswertbar zu gestalten, wird das Blitzlicht schriftlich erhoben. Dies ermöglicht auch, diese Evaluationstechnik in Gruppensettings anzuwenden. Zumal im Coaching das Schreiben Thema ist, reiht sich diese Qualitätsmaßnahme nahtlos in die schreibdidaktischen Interventionen ein.

Für die Rückmeldung werden selbsthaftende Notizblätter in Blumenform ${ }^{4}$ verteilt, auf welche die Coachees ihre Antworten schreiben sollen. Die Evaluation wird mehrfach durchgeführt - zu einzelnen Coachingterminen und am Ende der Durchführungsphase - und deckt mehrere Qualitätsdimensionen des Coachings ab. Um das Spektrum der erfassten Wahrnehmung nicht einzugrenzen, wird diese offen erfragt. Daher lautet die Anleitung zur Evaluation - immer gleich - wie folgt: „Bitte gib mir Feedback zum Schreibcoaching (eine Blume)“ und „Bitte gib mir Feedback zu mir als Person (eine Blume)“. Als zeitlicher Rahmen werden dafür maximal fünf Minuten für die Erstellung der Rückmeldungen angesetzt. Die Coachees werden gebeten, die beiden Blumen auf einem Blatt Papier (im Einzelcoaching) bzw. auf ein Flipchart (im Gruppencoaching) anzubringen, auf dem die Stängel eines Blumenstraußes vorgezeichnet sind.

Abhängig vom Setting, dem Coachingverlauf und dem aktuellen Stand des Schreibcoachings können die Feedbackblumen unmittelbar und gemeinsam mit den Coachees

\footnotetext{
${ }^{4}$ Was zur Namensgebung für diese Technik führte.
} 
besprochen werden, um auf den weiteren Coachingverlauf Einfluss zu nehmen oder in einem Reflexionsmoment außerhalb des Coachings ausgewertet werden. Die Evaluation wird von der Schreibcoachin mit abschließenden und verabschiedenden Worten „Danke für die Blumen“ beendet.

Es ist bewusst, dass diese Art der Evaluation das Ergebnis beeinflusst: So ist der Platz für Antworten physisch begrenzt $^{5}$, was dazu führt, dass Coachees in ihrer Antwort die am stärksten präsente Wahrnehmung notieren - sich also auf das Wesentliche fokussieren. Zum anderen kann Setting und Stimuli der Blumenform bzw. des Blumenstraußes eine Disposition zu positiver Rückmeldung erzeugen.

\subsection{Daten des Fallbeispiels}

Das vorgestellte Fallbeispiel beschränkt sich auf die für berufliches Schreiben durchgeführten Schreibcoachings und die Evaluationen in der Durchführungsphase bzw. an deren Ende. In den Jahren 2014-2020 wurden in diesem Tätigkeitsfeld 22 Schreibcoachings durchgeführt, der Datensatz umfasst 350 Blumen. Die Schreibcoachings fanden im Einzel- oder Gruppensettings statt und sind in die in Tab. 5 aufgeführten Auftragskategorien bzw. Aufgabenstellung des Schreibcoachings einzuteilen.

Die in diesen Schreibcoachings behandelten Texte und Problemstellungen sind sehr unterschiedlich, können jedoch anhand ihrer Textfunktion eingeteilt werden. Tab. 6 gibt eine Übersicht.

Die Teilnehmenden dieser beruflichen Schreibcoachings befinden sich in unterschiedlichen Positionen in ihrem Karriereweg, können aber in Angestellte oder Führungskräfte eingeteilt werden. Dies ist für die folgende Auswertung des Fallbeispiels dahingehend relevant, dass in einem Schreibgruppencoaching durchaus Angestellte und Führungskräfte zusammenkommen und sich gemeinsam über das Verfassen von Texten austauschen können.

Die Datensichtung und Analyse erfolgt in zwei Schritten: Die erste Datensichtung passiert direkt im Anschluss an die Erhebung - um daraus Rückschlüsse auf den weiteren Coachingverlauf abzuleiten. Die Notizblätter werden dann mit Kenndaten versehen (Auftrag, Auftraggeber*in, Datum) und archiviert. Die zweite (systematische) Datensichtung und Auswertung der Evaluationsrückmeldungen findet in definierten Intervallen statt (quartalsweise) und besteht aus der inhaltlichen Analyse (siehe Abschn. 5.5) und die darauf aufbauende statistische Analyse (siehe Abschn. 5.6). Die aus den Ergebnissen abzuleitenden Aktivitäten werden in Abschn. 5.7 beschrieben.

\footnotetext{
5 typische Größe der Notizblätter: $7 \times 7 \mathrm{~cm}$.
}

Tab. 5 Auftragskategorien im Schreibcoaching der Autorin

\begin{tabular}{|c|c|c|}
\hline $\begin{array}{l}\text { Kurzbezeich- } \\
\text { nung für } \\
\text { Auftragsart }\end{array}$ & Auftrag & $\begin{array}{l}\text { Bei der ersten Problembeschrei- } \\
\text { bung durch den Coachee würde } \\
\text { sich dies in Aussagen dieser Art } \\
\text { äußern: }\end{array}$ \\
\hline $\begin{array}{l}\text { Text- } \\
\text { kompetenz }\end{array}$ & $\begin{array}{l}\text { Text- } \\
\text { kompetenz } \\
\text { steigern }\end{array}$ & $\begin{array}{l}\text { „Die Berichte sind ,schlecht', sie } \\
\text { sollen , besser werden ““. } \\
\text { Auftrag in diesem Coaching ist, die } \\
\text { Textkompetenz des/der Schreiben- } \\
\text { den zu steigern }\end{array}$ \\
\hline $\begin{array}{l}\text { Schreib- } \\
\text { kompetenz }\end{array}$ & $\begin{array}{l}\text { Schreib- } \\
\text { kompetenz } \\
\text { steigern }\end{array}$ & $\begin{array}{l}\text { „Ich tue mir schwer beim Verfas- } \\
\text { sen von Protokollen, muss diese } \\
\text { aber so oft schreiben.“ } \\
\text { Auftrag in diesem Coaching ist, die } \\
\text { Schreibkompetenz zu steigern }\end{array}$ \\
\hline $\begin{array}{l}\text { Identifi- } \\
\text { kation }\end{array}$ & $\begin{array}{l}\text { Identifikation } \\
\text { des Schreib- } \\
\text { problems } \\
\text { durch das } \\
\text { Schreibcoa- } \\
\text { ching selbst }\end{array}$ & $\begin{array}{l}\text { „Ich habe den Eindruck, irgendet- } \\
\text { was passt nicht bei diesem Text, ich } \\
\text { weiß aber nicht genau was.“ } \\
\text { Der Auftrag in diesem Coaching } \\
\text { ist, ein Schreibproblem, eine Hem- } \\
\text { mung, eine Blockade zu identifi- } \\
\text { zieren und - wenn möglich - eine } \\
\text { passende Lösung zu erarbeiten }\end{array}$ \\
\hline Klarheit & $\begin{array}{l}\text { Klarheit } \\
\text { durch Schrei- } \\
\text { ben/durch } \\
\text { Text }\end{array}$ & $\begin{array}{l}\text { „Ich muss etwas über meine Ar- } \\
\text { beit, meine Leistung [o. Ä.] schrei- } \\
\text { ben, weiß aber nicht, wie ich das } \\
\text { ausdrücken soll.“ } \\
\text { Der Auftrag in diesem Coaching } \\
\text { ist, durch das Schreiben Klarheit } \\
\text { zu schaffen - Schreiben ist als das } \\
\text { Mittel, zu einem klaren, aussage- } \\
\text { kräftigen Text }\end{array}$ \\
\hline
\end{tabular}

Tab. 6 Texte und Textfunktionen im Schreibcoaching der Autorin

\begin{tabular}{ll}
\hline Textfunktion & Beispiele \\
\hline Informieren & $\begin{array}{l}\text { Berichte, Reports, Informationsblätter, Ausschreibun- } \\
\text { gen, Ankündigungen ... }\end{array}$ \\
$\begin{array}{ll}\text { Dokumen- } \\
\text { tieren }\end{array}$ & $\begin{array}{l}\text { Protokolle, Aktennotizen, Aufzeichnungen des Quali- } \\
\text { tätswesens ... }\end{array}$ \\
$\begin{array}{l}\text { Repräsen- } \\
\text { tieren }\end{array}$ & $\begin{array}{l}\text { Website-Texte, Social-Media-Beiträge, Leistungsdar- } \\
\text { stellungen im öffentlichen Raum, Leistungsankündi- } \\
\text { gungen für Zielgruppen ... }\end{array}$ \\
\hline
\end{tabular}

\subsection{Inhaltliche Auswertung der Evaluationen}

Die Evaluation der Rückmeldungen erfolgt mittels der inhaltlich strukturierenden, qualitativen Inhaltsanalyse nach Kuckartz (2012), welche auf das Identifizieren, Systematisieren und Analysieren von Themen und Subthemen abzielt. Die Kategorienbildung erfolgt in einer deduktiv-induktiven Mischform, was die Erweiterung/Änderung der verwendeten Kategorien ermöglicht - essenziell, um wiederkehrende Fremdwahrnehmung ins eigene Coachingkonzept einfließen lassen zu können. Die aus der Inhaltsanalyse emergierten Kategorien und Unterkategorien bilden die Qualitätsdimensionen des Coachingkonzepts und die Qualitätspolitik (siehe Abschn. 5.1) gut ab. Sie wurden im Laufe der Jahre auf Basis der Weiterentwicklung des Qualitätskonzepts 
Tab. 7 Kategoriensystem zur Auswertung der erhobenen Evaluationsdaten

\begin{tabular}{|c|c|c|}
\hline Oberkategorie & $\begin{array}{l}\text { Unterkategorie, } \\
\text { Kurzbezeichnung }\end{array}$ & Unterkategorie: Die Rückmeldung beinhaltet eine Aussage zu \\
\hline \multirow{5}{*}{$\begin{array}{l}\text { Ergebnis } \\
\text { bezieht sich auf die } \\
\text { Ergebnisqualität des } \\
\text { Schreibcoachings }\end{array}$} & Ziel & $\begin{array}{l}\text { Zielerreichung, Erfüllung der Erwartungen in Bezug auf Ergebnis bzw. Verlauf, } \\
\text { die Intervention(en) waren hilfreich, nützlich, erfolgreich }\end{array}$ \\
\hline & Lernzuwachs & Ich habe etwas gelernt, ich nehme etwas mit \\
\hline & Erwartung & Die Erwartungen wurden nicht nur erfüllt, sondern übertroffen \\
\hline & Klärend & Das Coaching brachte Klarheit, wirkte klärend \\
\hline & Wiederbeauftragung & Der Wunsch nach Wiederbeauftragung für ein anderes Feld wird explizit genannt \\
\hline \multirow{2}{*}{$\begin{array}{l}\text { Prozess } \\
\text { bezieht sich auf die } \\
\text { Prozessqualität des } \\
\text { Schreibcoachings }\end{array}$} & Strukturiert & Der Verlauf des Coachings wird als strukturiert beschrieben \\
\hline & Durchdacht & Der Verlauf wird als vorbereitet, organisiert, durchdacht beschrieben \\
\hline \multirow{3}{*}{$\begin{array}{l}\text { Interaktion } \\
\text { bezieht sich auf die Merkmale } \\
\text { der Interaktion und der } \\
\text { Beziehung }\end{array}$} & Wertschätzend & Der Umgang im Schreibcoaching wird als wertschätzend beschreiben \\
\hline & Motivierend & Die Interaktion wird impulsgebend, motivierend, inspirierend \\
\hline & Humor & $\begin{array}{l}\text { In den Rückmeldungen wird Bezug auf eine humorvolle Situation genommen, die } \\
\text { nur die am Schreibcoaching beteiligten Personen verstehen („Insider-Scherz“) }\end{array}$ \\
\hline \multirow{3}{*}{$\begin{array}{l}\text { Kompetenz } \\
\text { bezieht sich auf die } \\
\text { Kompetenz der Coachin }\end{array}$} & Methodenkompetenz & Die Methodik des Coachings wird beschrieben \\
\hline & Fachkompetenz & Der fachliche Input im Coaching, die Fachkompetenz der Coachin wird angeführt \\
\hline & Feldkompetenz & $\begin{array}{l}\text { Die Kompetenz im Schreiben (z.B. Schreibtechniken, Schreibmethoden) werden } \\
\text { angeführt }\end{array}$ \\
\hline \multirow{3}{*}{$\begin{array}{l}\text { Person } \\
\text { bezieht sich auf } \\
\text { Persönlichkeitsmerkmale, } \\
\text { Eigenschaften der Coachin }\end{array}$} & Präzise & Der Ausdruck, die Wortwahl der Coachin wird als präzise beschrieben \\
\hline & Persönlichkeit & Eine persönliche Eigenschaft der Coachin wird erwähnt (z. B. charmant) \\
\hline & Auftreten & $\begin{array}{l}\text { Das Auftreten der Coachin wird kommentiert, z. B. „,cooler Hut“ oder ,professio- } \\
\text { nelles Auftreten“ }\end{array}$ \\
\hline Visualisierung & Visualisierung & Die Rückmeldung enthält eine Zeichnung, ein Emoticon, eine Visualisierung \\
\hline Danke & Danke & $\begin{array}{l}\text { Positive Rückmeldung generischer Art, Dankesworte, verbunden mit einer Art } \\
\text { von Lob, z. B.: „Danke, es war super!“” }\end{array}$ \\
\hline Kritik & Kritik & Kritische Wortäußerung, Rückmeldung zu einem (noch) fehlenden Aspekt \\
\hline
\end{tabular}

bzw. der Rückmeldungen im Coaching erweitert/adaptiert. Tab. 7 zeigt den aktuellen Stand (Mai 2020).

Die inhaltsanalytische Auswertung zeigt, dass die Antworten auf die zwei Fragen - Rückmeldung zum Schreibcoaching, Rückmeldung zur Schreibcoachin - in allen Kategorien zu verorten sind. Die ursprünglich erhoffte Differenzierung (und damit erleichterte inhaltliche Auswertung in Person und Coaching) kann aus den Antworten nicht abgelesen werden.

\subsection{Statistische Auswertung der Evaluationen}

Die klassifizierten Daten aus Abschn. 5.5 werden in Excel festgehalten und für die in Folge stattfindende statistische Auswertung in die Statistiksoftware R importiert. Hier soll exemplarisch eine statistische Auswertung für drei Zielfragen im Rahmen des Qualitätsmanagements aufgeführt werden: Welche Wahrnehmung haben unterschiedliche Zielgruppen? Hat die Auftragsart (vgl. Tab. 5) Einfluss auf das Coaching? Und: Hat die Textfunktion (vgl. Tab. 6) Auswirkung auf die Wahrnehmung der Coachingqualität?

\subsubsection{Allgemeine statistische Kennzahlen des Datensatzes}

In den 22 Coachings gab es im Schnitt 15,91 (sd=9,65) Blumen je Coaching, welche in bzw. am Ende der Durchführungsphase erhoben wurden. Von den 350 Blumen wurden 184 Blumen zur Person der Coachin und 166 Blumen zum Coaching verfasst. Im Durchschnitt enthält jede Blume 4,92 (sd=4,09) Wörter und 35,4 (sd=27,3) Zeichen. Eine signifikante Differenz zwischen Blumen zur Person der Coachin und Blumen fürs Coaching ist nicht gegeben (Wörterdifferenz: $\mathrm{CI}^{95}=[-0,52 \ldots 1,19], p=0,44$; Zeichendifferenz: $\left.\mathrm{CI}^{95}=[-4,20 \ldots 7,28], p=0,60\right)$.

Nach der Zuordnung der Blumentexte zu den Kategorien (Mehrfachzuordnung möglich), zeigen sich die Korrelation in Tab. 8. Es gibt deutliche Häufigkeitsunterschiede zwischen den Kategorien $\left(\xi^{2}=352\right.$, df $\left.=15, p<0,001\right)$. Auffällig ist die Verteilung der Korrelationen: Rückmeldungen nehmen sich gegenseitig Platz auf der Blume weg (deshalb überwiegend negative Korrelation) bzw. Wortpaare bzw. Phrasenpaare ergeben positive Korrelation (Beispiel: Korrelation zwischen Persönlichkeit und Auftreten).

In Summe gibt es 10 Blumen mit Kritik, davon 5, die ausschließlich Kritik enthalten. 90 Blumen enthielten eine generische positive Rückmeldung (Danke), davon 32 
Tab. 8 Korrelation (Kendalls $\tau)$ der Kategorien $(n=350)$

\begin{tabular}{|c|c|c|c|c|c|c|c|c|c|c|c|c|c|c|c|c|}
\hline Kategorie & $n$ & 1 & 2 & 3 & 4 & 5 & 6 & 7 & 8 & 9 & 10 & 11 & 12 & 13 & 14 & 15 \\
\hline $1 \mathrm{Ziel}$ & 41 & - & - & - & - & - & - & - & - & - & - & - & - & - & - & - \\
\hline $\begin{array}{l}2 \text { Lernzu- } \\
\text { wachs }\end{array}$ & 19 & 0,070 & - & - & - & - & - & - & - & - & - & - & - & - & - & - \\
\hline 3 Erwartung & 7 & 0,011 & $-0,034$ & - & - & - & - & - & - & - & - & - & - & - & - & - \\
\hline 4 Klärend & 19 & 0,030 & 0,054 & $-0,034$ & - & - & - & - & - & - & - & - & - & - & - & - \\
\hline $\begin{array}{l}5 \text { Wiederbe- } \\
\text { auftragung }\end{array}$ & 11 & 0,036 & $-0,043$ & 0,091 & $-0,043$ & - & - & - & - & - & - & - & - & - & - & - \\
\hline 6 Strukturiert & 48 & $-0,094$ & 0,051 & 0,062 & 0,014 & $-0,072$ & - & - & - & - & - & - & - & - & - & - \\
\hline 7 Durchdacht & 25 & $-0,101$ & $-0,066$ & 0,040 & $-0,066$ & $-0,050$ & $0,115^{*}$ & - & - & - & - & - & - & - & - & - \\
\hline $\begin{array}{l}8 \text { Wertschät- } \\
\text { zend }\end{array}$ & 9 & $-0,059$ & $-0,039$ & $-0,023$ & $-0,039$ & $-0,029$ & $-0,012$ & $-0,045$ & - & - & - & - & - & - & - & - \\
\hline 9 Motivierend & 32 & $-0,023$ & $-0,076$ & 0,025 & 0,055 & 0,000 & $-0,011$ & $-0,011$ & 0,074 & - & - & - & - & - & - & - \\
\hline 10 Humor & 6 & $-0,048$ & $-0,032$ & $-0,019$ & $-0,032$ & $-0,024$ & 0,075 & 0,049 & $-0,021$ & $-0,042$ & - & - & - & - & - & - \\
\hline $\begin{array}{l}11 \text { Methoden- } \\
\text { kompetenz }\end{array}$ & 108 & $-0,089$ & $-0,078$ & $-0,051$ & $-0,078$ & $-0,014$ & 0,021 & 0,031 & $-0,030$ & $-0,040$ & $-0,041$ & - & - & - & - & - \\
\hline $\begin{array}{l}12 \text { Fachkom- } \\
\text { petenz }\end{array}$ & 61 & $-0,027$ & $-0,010$ & 0,150 ** & $-0,044$ & 0,047 & $-0,030$ & 0,019 & $-0,075$ & $-0,067$ & $-0,061$ & $-0,062$ & - & - & - & - \\
\hline $\begin{array}{l}13 \text { Feldkom- } \\
\text { petenz }\end{array}$ & 43 & $-0,109 *$ & $-0,013$ & $-0,053$ & $-0,090$ & $-0,067$ & $-0,099$ & $-0,036$ & 0,104 & $-0,089$ & $-0,049$ & $0,165^{* *}$ & $-0,126^{*}$ & - & - & - \\
\hline 14 Präzise & 12 & $-0,069$ & 0,024 & $-0,027$ & 0,024 & $-0,034$ & 0,062 & 0,009 & $-0,031$ & $-0,060$ & $-0,025$ & $0,112 *$ & $-0,045$ & 0,025 & - & - \\
\hline $\begin{array}{l}15 \text { Persönlich- } \\
\text { keit }\end{array}$ & 51 & $-0,150^{* *}$ & $-0,063$ & $-0,059$ & $-0,063$ & $-0,074$ & $-0,070$ & $-0,020$ & $0,138^{*}$ & $-0,019$ & 0,008 & 0,057 & $-0,147 * *$ & 0,018 & 0,100 & - \\
\hline 16 Auftreten & 34 & $-0,119^{*}$ & $-0,079$ & $-0,047$ & $-0,079$ & $-0,059$ & 0,009 & $-0,054$ & 0,069 & 0,030 & $-0,043$ & $-0,031$ & $-0,100$ & 0,054 & 0,044 & $0,165^{* *}$ \\
\hline
\end{tabular}

$* p<0,05 ; * * p<0,01$

Tab. 9 Korrelation (Kendalls $\tau$ ) der Oberkategorien $(n=312)$

\begin{tabular}{llllll}
\hline Kategorie & $n$ & 1 & 2 & 3 & 4 \\
\hline 1 Ergebnis & 85 & - & - & - & - \\
2 Prozess & 66 & $-0,086$ & - & - & - \\
3 Interaktion & 45 & $-0,098$ & 0,033 & - & - \\
4 Kompetenz & 175 & $-0,180^{* * *}$ & $-0,044$ & $-0,128^{*}$ & - \\
5 Person & 82 & $-0,250^{* * *}$ & $-0,042$ & 0,050 & $-0,040$ \\
\hline
\end{tabular}

$* p<0,05 ; * * p<0,01 ; * * * p<0,001$ ausschließlich diese Rückmeldung. Da kritische Blumen in zu geringer Anzahl vorliegen, um sinnvolle Aussagen zu treffen, und auch generisch-positive Blumen keine Zuordnung zu den Oberkategorien erlauben, wurden 38 Blumen für die weitere Analyse nicht berücksichtigt $(5 \times$ nur Kritik, $32 \times$ nur generisch-positiv, $1 \times$ Kritik + generisch-positive Rückmeldung). Zusätzlich zu den verbalen Rückmeldungen sind auf 27 Blumen visuelle Darstellungen vorhanden, welche für die statistische Auswertung ebenfalls unberücksichtigt bleiben.

Nach der Zuordnung der einzelnen Kategorien zu den Oberkategorien ergibt sich die Verteilung in Tab. 9. Auch hier gibt es eine deutliche Ungleichverteilung $\left(\xi^{2}=147,6\right.$; $\mathrm{df}=4 ; p<0,001)$ der Oberkategorien. Wiederum sind (fast) alle Korrelationen negativ. Während sich bei den Einzelkategorien noch Phrasen und Wortpaare finden, die auf mehrere Kategorien zutreffen, gilt das für Oberkategorien nicht mehr.
Auf eine mathematische Prüfung der Konstruktion der Oberkategorien durch die Kategorien (Cronbachs Alpha, Kaiser-Meyer-Olkin etc.) wurde bewusst verzichtet. Zum einen sind die Kategorien keine Skalen im statistischen Sinn, zum anderen beeinflussen sich die Kategorien derselben Oberkategorie durch die Platzknappheit auf den Blumen negativ: Wird bspw. etwas zur Fachkompetenz notiert, wird häufig die Feldkompetenz nicht erwähnt. Dementsprechend und weil es sich ausschließlich um positive Rückmeldungen handelt, muss auch das Ergebnis interpretiert werden: Die häufigere Rückmeldung von z. B. Kompetenz (175) im Vergleich zu Ergebnis (85) bedeutet nicht, dass die Coachin kompetenter ist als es das Ergebnis des Coachings vermuten lässt. Stattdessen spiegeln die Häufigkeiten die zum Zeitpunkt der Erhebung dominante Coachee-Wahrnehmung des Coachings bzw. der Coachin wider. Aus der deutlich negativen Korrelation von Kompetenz und Person gegenüber Ergebnis kann daher geschlossen werden, dass abhängig von den Coachingzielen unterschiedliche Wahrnehmungen 
dominieren, denn es gibt keinen sachlichen Grund, dass eine als kompetent wahrgenommene Coachin schlechtere Ergebnisse erzielt.

\subsubsection{Einfluss der Auftragsart auf das Coaching}

Bei der Unterscheidung der Oberkategorien nach Auftragsart (Abb. 2) fallen markante Unterschiede in der Qualitätswahrnehmung auf. Am Beispiel der Auftragsart Textkompetenz steigern zeigt sich, dass Rückmeldungen zur Prozessqualität des Coachings weniger häufig angeführt werden als in Coachings der Auftragsart Steigerung der Schreibkompetenz (Effektgröße $\mathrm{h}=0,50^{* *} ; \xi^{2}=9,92$; $\mathrm{df}=1$ ). Im Vergleich zu Coachings der Auftragsart Identifikation des Schreibproblems lässt sich bei Textkompetenz steigern - durch den Fokus auf die im Coaching entstehenden Texte - auch das Ergebnis besser einschätzen. Dementsprechend höher ist die Wahrnehmung der Ergebnisqualität (Effektgröße $\mathrm{h}=0,67^{* * *} ; \xi^{2}=14,1 ; \mathrm{df}=1$ ).

\subsubsection{Wahrnehmung durch unterschiedliche Zielgruppen}

Werden Oberkategorien nach Zielgruppe unterschieden (Abb. 3), zeigt sich auch hier ein Unterschied in der Ergebniswahrnehmung zwischen Angestellten und Führungskräften, der mit einer Effektgröße von $\mathrm{h}=0,45^{* *}\left(\xi^{2}=9,26\right.$; $\mathrm{df}=1)$ deutlich ausfällt. Der Unterschied in der Wahrnehmung der Prozessqualität ist insofern interessant, als dass die getrennten Zielgruppen eine ähnliche Wahrnehmung haben; Bei Gruppencoachings, an denen beide Zielgruppen teilnehmen, aber die Prozessqualität deutlich häufiger Thema ist (gemittelte Effektgröße $\mathrm{h}=0,45^{* *} ; \xi^{2}=12,26$; $\mathrm{df}=2$ ). Es liegt der Schluss nahe, dass hier im Coaching wirklich die Prozessdimension wichtiger ist.

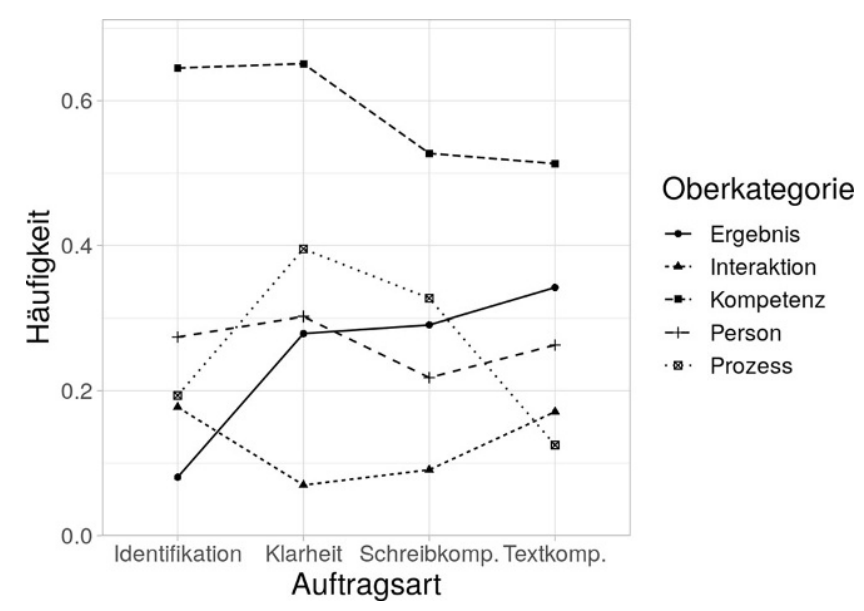

Abb. 2 Häufigkeit der Oberkategorien nach Auftragsart

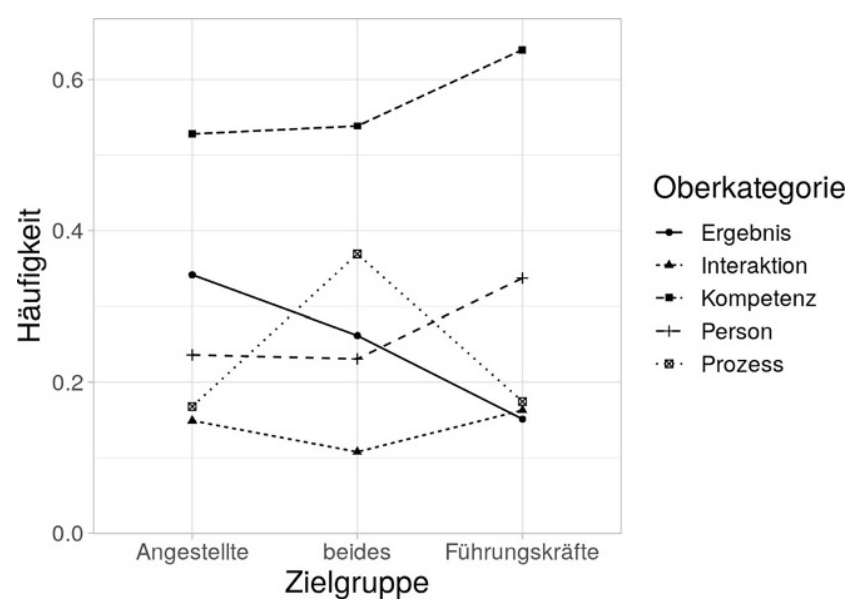

Abb. 3 Häufigkeit der Oberkategorien je Zielgruppe

\subsubsection{Einfluss der Textfunktion}

Werden die Oberkategorien nach Textfunktion aufgeteilt, fallen die Unterschiede geringer aus (Abb. 4). Die Häufigkeit der Qualitätswahrnehmung des Coachingergebnisses hat abhängig von der Textfunktion Dokumentieren/ Informieren oder Repräsentieren eine gemittelte EffektgröBe $\mathrm{h}=0,25\left(\xi^{2}=3,89 ; \mathrm{df}=2\right)$, die anderen Qualitätsdimensionen werden annähernd identisch wahrgenommen.

\subsection{Auswirkung und Rückfluss ins Qualitätsmanagement}

Die vorangegangenen Ergebnisse der statistischen Analyse fließen im Sinne des in Abschn. 5.1 vorgestellten PDCAZyklus wieder in die schreibdidaktische Arbeit der Autorin rück. Sie werden im Rahmen einer Managementbewertung in einer individuell gestalteten Balanced Scorecard zusammengeführt und analysiert und mit den Qualitätskriterien

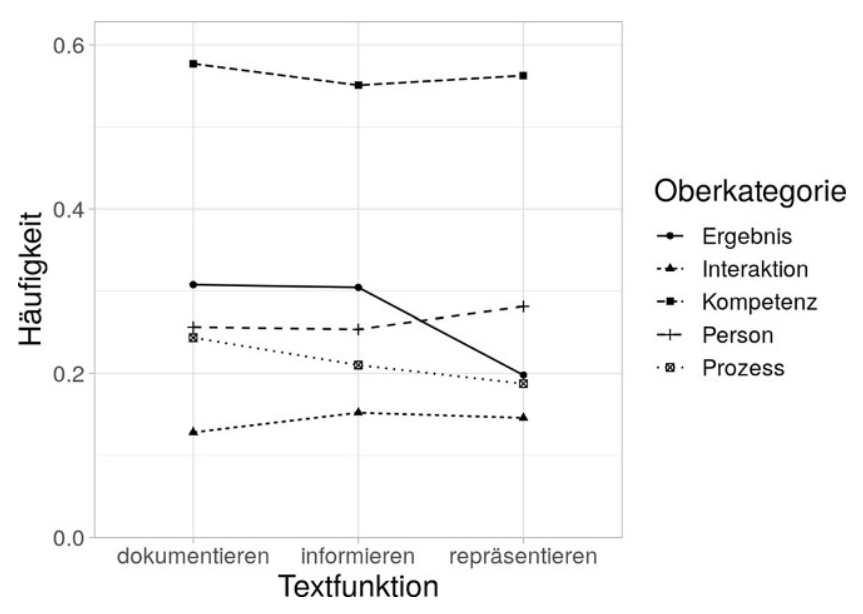

Abb. 4 Häufigkeit der Oberkategorien je Textfunktion 
abgeglichen. Diese Managementbewertung resultiert in Fragen nach dem Wie der kontinuierlichen Verbesserung, was nachstehend exemplarisch gezeigt wird.

Unterschiede können etwa durch die eingehende Analyse der Ergebnisse differenziert und erklärt werden, ohne Handlungsbedarf auszulösen. Wie in Abb. 2 visualisiert, gibt es nach Auftragsart unterschiedlich häufige Rückmeldungen zur Prozessqualität. Während in Coachings zur Steigerung der Schreibkompetenz schreibprozessorientiert gearbeitet wird, Prozesse also kontinuierlich thematisiert, analysiert, visualisiert werden, wird bei Textkompetenz-Coachings produkt- und textsortenorientiert gearbeitet, Prozesse werden also weniger thematisiert. Analog erklären sich die Häufigkeitsunterschiede in der Wahrnehmung der Ergebnisqualität: Bei der Auftragsart Textkompetenz steigern steht das Ergebnis, das fertige Textprodukt im Vordergrund. Dieser Fokus führt in Folge zu einer häufigeren Wahrnehmung - anders als bei Coachingaufträgen, bei denen es um die Identifikation von Schreibproblemen geht: Hier war der Fokus auf die Kompetenz der Coachin, das Problem erkannt und eine entsprechende Lösung angeboten zu haben. In beiden Fällen ergibt die eingehende Analyse der Ergebnisse also keine weitere Handlungsnotwendigkeit.

Die Analyse der Wahrnehmungsrückmeldungen nach Zielgruppe (Abb. 3) lässt sich schreibdidaktisch jedoch nicht erklären. Im Vergleich der Zielgruppen fällt auf, dass sich die Wahrnehmungen bei Führungskräften hinsichtlich der Ergebnisqualität deutlich von jenen der Angestellten unterscheiden. Dies ist vor allem vor dem Hintergrund der sich gegenteilig darstellenden Kompetenzwahrnehmung spannend. Die Managementbewertung, in der neben der Blumenevaluation auch weitere Key Performance Indicators und persönliche Reflexionsaufzeichnungen herangezogen wurden, führte zu Nachgesprächen mit einigen Führungskräften-Coachees, um die Ergebnisqualität im Detail zu beleuchten. Die differenzierte Analyse offenbarte Verbesserungspotentiale im Bereich der Feldkompetenz der Coachin, was zu einer Fortbildungsmaßnahme (zum Thema Management-Review) und einem vertiefenden Literaturstudium (zum Thema Management-Reporting) führte, um die Welt der Führungskräfte und ihre Anforderungen an Texte noch besser verstehen zu können ${ }^{6}$. Zugleich führten diese Reflexionsgespräche auf Seiten der Führungskräfte zu einer noch stärkeren Bewusstwerdung der organisationsspezifischen Eigenheiten und der daraus resultierenden Notwendigkeit der Offenlegung der Wünsche und Anforderungen der Führungskräfte. Dieser Punkt wird nun auch explizit in der Vorphase eines Coachings aktiv von der Coachin abgefragt.

\footnotetext{
${ }^{6}$ Qualitätskriterien: Empathie, Feldkompetenz in Domänen - vgl.
} Qualitätspolitik in Abschn. 5.1.
Am Beispiel der Abb. 4 zur Qualitätswahrnehmungen nach Textfunktion lassen sich auch andere Verbesserungsmaßnahmen aufzeigen: Hier sind Wahrnehmungsunterschiede in Bezug auf die Ergebnisqualität im Vergleich der Textfunktionen dokumentieren und repräsentieren erkennbar. Um Coachees noch mehr Hilfe zur Selbsthilfe (siehe dazu Qualitätspolitik in Abschn. 5.1) ermöglichen zu können, und somit die Ergebnisqualität zu steigern, ist es von Seiten der Coachin nötig, Texte der Textfunktion repräsentieren ${ }^{7}$ gut zu kennen und auch ,am Puls der Zeit“" zu sein. Statt Weiterbildung (welche sich vorwiegend an Domänenexpert*innen richtet) holt die Autorin in facheinschlägigen Treffen und in branchenübergreifenden Netzwerktreffen die Expertise von Betroffenen ein und transferiert diese synektisch ${ }^{8}$ auf Coachings zu repräsentativen Textsorten.

\section{Diskussion}

Das Fallbeispiel belegt, dass es möglich ist, als einzelne*r Schreibcoach*in ein Qualitätsmanagementsystem zu implementieren, dessen Form, Medium und Durchführung auf die Coachingssituation und den*die Coach*in so adaptiert wird, dass es stimmig zum Coaching-Design sowie zur Person des*der Coaches*in ist, die Messung selbst sich nahtlos an die Schreibinterventionen anschließt und dennoch wertvolle Aussagen zur wahrgenommen Qualität gewonnen werden und Handlungsanforderungen abgeleitet werden können.

Die dargestellten Ergebnisse der Schreibcoachings aus dem Bezugsrahmen des beruflichen Schreibens zeigen, dass die offene Fragestellung der eingesetzten Feedbacktechnik Danke für die Blumen geeignet ist, die unmittelbare Wahrnehmung zu erheben und dass diese alle Qualitätsdimensionen aufweist (Prozessqualität, Ergebnisqualität, Personenqualität). Betrachtet man diese Rückmeldungen auf einer Metaebene, wird ein Bild der Dienstleistung des Schreibcoachings und des Schreibcoach gezeichnet, das nahe dem Qualitätskonzept der Autorin ist. Dies wiederum lässt Rückschlüsse auf die Passungsqualität zu, die in der Vorphase des Schreibcoachings als Quality-Gate zur Entscheidung über die Durchführung des Schreibcoachings fungiert (vgl. Hanke und Boehnke 2018). Zudem zeigt die systematische Auswertung Aspekte auf, die im punktuellen Feedback nicht unmittelbar ersichtlich sind, beispielsweise die unterschiedliche Qualitätswahrnehmung je Auftragsart bzw. je Zielgruppe.

\footnotetext{
7 Websites, Social-Media-Beiträge, Jahresberichte, Broschüren etc.

${ }^{8}$ Die Synektik ist eine Kreativitätstechnik von Gordon (1961) um mittels Transfer (fach-)fremden Wissens Probleme zu lösen.
} 
Notwendige Basis dafür sind das Coaching-Leitbild und das Verständnis von Coaching als steten, kontinuierlich zu verbessernden Prozess (vgl. Schmidt-Lellek 2006; und Steinke 2010). Diese Iteration erlaubt es, systematisch Evaluationsergebnisse zu denselben Messzeitpunkten und mit demselben Messinstrument zu sammeln. Das Leitbild bildet die Grundlage für die Kategorienbildung der Qualitätsdimensionen, welche im Fallbeispiel im Sinne des BC-Modells (Brady und Cronin 2001) hierarchisch operationalisiert und in Folge der laufenden Auswertung (den Blumen) auf Basis der qualitativen Inhaltsanalyse nach Kuckartz (2012) erweitert bzw. abgeändert wurden.

Eine zeitliche Entwicklung und Veränderung könnte ebenfalls aus den Ergebnissen abgelesen werden und so dazu beitragen, Potenzial für eigene Weiterbildung zu entdecken (vgl. Schermuly und Graßmann 2015; Lippmann 2015; sowie Abschn. 3.1) bzw. zu prüfen, ob deren Auswirkung sichtbar wird. Aus den Daten des Fallbeispiels allein lassen sich aber derzeit noch keine aussagekräftigen kausalen Schlüsse ziehen.

\subsection{Grenzen der Studie}

Die Studie kann ob ihrer Natur nur bedingt verallgemeinert werden: Sie zeigt im Fallbeispiel einen Ausschnitt des schreibdidaktischen Leistungsspektrums nur einer Schreibcoachin (der Autorin). Das Modell muss daher für jede*n Schreibcoach*in adaptiert werden, bzw. jede Schreibcoach*in sollte (entsprechend den Forderungen von Steinke 2010) überlegen, welche Ausprägung das eigene Qualitätskonzept hat. Die Ergebnisse der Evaluationen der Autorin belegen aber beispielhaft, welche Informationen und Handlungs- bzw. Verbesserungsimpulse aus dem vorgestellten Modell zum Qualitätsmanagementsystem gezogen werden können.

Das Messinstrument (Evaluationsfragen) und sein angemessener Einsatz spielen eine gewichtige Rolle. Es ist nicht davon auszugehen, dass die Blumenform für andere Coach*innen passend ist, während das Blitzlicht-Feedback selbst durchaus in Betracht gezogen werden sollte. Auch der Zeitpunkt der Evaluation ist vor dem Hintergrund des Ziels der Qualitätsmessung zu betrachten: Sollen unmittelbare Wahrnehmungen, Einflüsse oder Ergebnisse gemessen werden? Oder sollen bereits reflektierte Gedanken abgefragt bzw. gesammelt werden? Die deutlich negative Korrelation der Kategorien und die geringe Wortanzahl je Feedback im Fallbeispiel weisen darauf hin, dass das gewählte Instrument (Blitzlicht in Form von Blumen) nur die zum Zeitpunkt der Erhebung dominierende Wahrnehmung erfassen kann. Zudem evoziert das Setting überwiegend positive Rückmeldungen. Dennoch können wertvolle Informationen und Rückschlüsse gezogen werden, wie in den Abschn. 5.6 und 5.7 dargelegt wird. Begleitende Maß- nahmen (bspw. punktuelle vertiefende Reflexionsgespräche, Mentoring oder Supervision) können zudem etwaige Messlücken schließen. Auf die Wichtigkeit der Weiterbildung sei in diesem Zusammenhang nochmal hingewiesen (vgl. Abschn. 3.1).

Das Fallbeispiel liefert auch keine Aussage über die Effektivität alternativer Interventionen oder begleitender Maßnahmen. Hier könnte weitere Forschung ansetzen. Auch alternative Messmethoden, deren Einsatztauglichkeit und Effektivität wurden im Fallbeispiel nicht beleuchtet. Inwiefern ressourcenintensivere summative Evaluierungsmethoden für den Einsatz im Coaching bzw. für Einpersonenunternehmen geeignet sind - Stichwort „Feedbackermüdung“ (Dainton 2018, S. 23) - könnten weitere wissenschaftliche Untersuchungen aufzeigen.

\section{Schlussfolgerung und Ausblick}

Der Qualitätsbegriff und dessen Messung wurde in der Schreibcoaching-Literatur bisher vor allem aus dem Blickwinkel des*der Coachee behandelt. Die vorliegende Studie beschreibt ein Modell zum Qualitätsmanagement für den*die Coach*in, dessen Umsetzbarkeit und Informationsgehalt anhand des Fallbeispiels Danke für die Blumen belegt worden ist. Das Fallbeispiel zeigt zudem das Potenzial für kurz- und mittelfristige Analysen auf, sofern eine systematische Erhebung stattfindet. Auch Steinke (2010) unterstreicht, dass ein Qualitätsmanagementsystem Reflexionsprozesse auslöse und die Kompetenzdarstellung erleichtere.

Diese kontinuierliche Qualitätsprüfung führt bereits innerhalb eines Coachings zu einer Qualitätssicherung - sowohl in Hinblick auf Prozess als auch auf das Ergebnis. Die abschließende Evaluation gibt Aufschluss auf den Prozessverlauf und die Wirksamkeit und ermöglicht zum einen auch dem*der Coachee eine Reflexion des Prozesses und des Ergebnisses, was wiederum zu einem bewussteren Verständnis des im Coaching Erarbeiteten führt. Zum anderen ermöglicht die abschließende Qualitätskontrolle eine tiefergehende Reflexion, die evidenzbasiert zu einer Weiterentwicklung führt.

Das Fallbeispiel zeigt, wie mit einfachen Fragen umfassende Rückmeldungen eingeholt werden können. Ein schriftlich durchgeführtes Blitzlicht-Feedback ist eine rasch umzusetzende und auch für Gruppensettings geeignete Form, flächendeckend und kontinuierlich Rückmeldungen zu erheben. Eine anschließende inhaltliche Auswertung legt offen, wie welche Qualitätsdimensionen des Schreibcoachings wahrgenommen und rückgemeldet werden.

Weitere Forschung sollte darauf fokussieren, welche Feedback-Methoden und -Techniken sowie welche Feedbackfragen besonders für das Schreibcoaching geeignet 
sind. Ebenso ist anzusetzen, welche Werte bzw. Grundhaltungen ein Qualitätskonzept für Schreibcoaching beinhalten sollte und welche Qualitätsdimensionen im Schreibcoaching im Gegensatz zum Coaching allgemein eine Rolle spielen. Zudem ist zu überlegen, ob das vorgestellte Modell des Qualitätsmanagementsystems auch für andere Formen der schreibdidaktischen Intervention bzw. Schreiblehrsituationen (Schreibtraining, Schreibberatung, schreibintensive Lehre, Schreiblehre ...) geeignet ist bzw. sich für Coachings mit anderen Schwerpunkten eignet.

Kritisch anzumerken ist, dass ein bloßes Vorhandensein von Qualitätsstandards nicht automatisch zu einer Qualitätsverbesserung des Schreibcoachings selbst führt. Welche Rahmenbedingungen dazu notwendig sind, ist eine offene Frage. Das vorliegende Modell zum Qualitätsmanagementsystem für das Schreibcoaching nimmt für sich in Anspruch, einen Baustein für diesen Diskurs zu liefern.

Danksagung Ich danke Theresa Boiger für die Unterstützung bei der Datensichtung und -verwaltung und für ihre klugen Überlegungen zur Systematik der Feedbackblumen. Mein besonderer Dank gilt Arno Hollosi für die hilfreichen Kommentare, konstruktiven Vorschläge und das scharfsichtige Betrachten der statistischen Ausführungen.

Open Access Dieser Artikel wird unter der Creative Commons Namensnennung 4.0 International Lizenz veröffentlicht, welche die Nutzung, Vervielfältigung, Bearbeitung, Verbreitung und Wiedergabe in jeglichem Medium und Format erlaubt, sofern Sie den/die ursprünglichen Autor(en) und die Quelle ordnungsgemäß nennen, einen Link zur Creative Commons Lizenz beifügen und angeben, ob Änderungen vorgenommen wurden.

Die in diesem Artikel enthaltenen Bilder und sonstiges Drittmaterial unterliegen ebenfalls der genannten Creative Commons Lizenz, sofern sich aus der Abbildungslegende nichts anderes ergibt. Sofern das betreffende Material nicht unter der genannten Creative Commons Lizenz steht und die betreffende Handlung nicht nach gesetzlichen Vorschriften erlaubt ist, ist für die oben aufgeführten Weiterverwendungen des Materials die Einwilligung des jeweiligen Rechteinhabers einzuholen.

Weitere Details zur Lizenz entnehmen Sie bitte der Lizenzinformation auf http://creativecommons.org/licenses/by/4.0/deed.de.

\section{Literatur}

Anders, S. (2002). Qualitätsmanagement und Qualitätssicherung im Coaching: konzeptionelle und inhaltliche Überlegungen zur Qualität der Einzelberatung von Führungskräften. Organisationsberatung Supervision Coaching, 9(2), 101-118. https://doi.org/10. 1007/s11613-002-0012-0.

Arnold, R. (1999). Qualität ist viereckig - Reflexionen zum Umgang mit Qualität in der Weiterbildung. PÄD Forum, 1, 35-38.

Behrendt, P. (2012). Freiburger Erfolgsfaktoren-Coaching. Vier Erfolgsfaktoren zur Etablierung von Konsistenz bei Coachees. Organisationsberatung Supervision Coaching, 19(4), 391-404. https://doi.org/10.1007/s11613-012-0296-7.

Berninger-Schäfer, E. (2018). Online-Coaching. Wiesbaden: Springer. https://doi.org/10.1007/978-3-658-10128-2.

Bortz, J., \& Döring, N. (2006). Besonderheiten der Evaluationsforschung. Forschungsmethoden und Evaluation. Springer-Lehrbuch. Berlin, Heidelberg: Springer. https://doi.org/10.1007/9783-540-33306-7_3.
Brady, M. K., \& Cronin Jr., J. J. (2001). Some new thoughts on conceptualizing perceived service quality: a hierarchical approach. Journal of Marketing, 65(3), 34-49. https://doi.org/10.1509/jmkg.65. 3.34.18334.

Brandsen, T., Steen, T., \& Verschuere, B. (Hrsg.). (2018). Co-production and co-creation: engaging citizens in public services. New York: Routledge.

Bräuer, G. (2014). Grundprinzipien der Schreibberatung. Eine pragmatische Sicht auf die Schreibprozesstheorie. In S. Dreyfürst \& N. Sennewald (Hrsg.), Schreiben. Grundlagentexte zur Theorie, Didaktik und Beratung (S. 257-282). Opladen Toronto: Budrich.

Brinkschulte, M., Grieshammer, E., \& Kreitz, D. (2014). Alles Roger(s)? Psychologische Ansätze für die Schreibberatung. Journal der Schreibberatung, 8, 1-12. https://doi.org/10.3278/ JOS1401W.

Bueno, E.V., Weber, T.B.B., Bomfim, E.L., \& Kato, H.T. (2019). Measuring customer experience in service: a systematic review. The Service Industries Journal, 39(11-12), 779-798. https://doi. org/10.1080/02642069.2018.1561873.

Chirico, R., Kollak, I., \& Lahmann, N. (2013a). Das Ganze ist mehr als die Summe seiner Teile: Die Kombination von Gruppen- und Einzel-Coaching. Journal der Schreibberatung, 6, 23-32.

Chirico, R., Kollak, I., \& Lahmann, N. (2013b). „Hat mir sehr geholfen!“ Ratsuchende evaluieren ein Schreibcoachingprogramm. Journal der Schreibberatung, 7, 11-22.

Cohn, R.C. (1975). Von der Psychoanalyse zur Themenzentrierten Interaktion. Von der Behandlung einzelner zu einer Pädagogik für alle. Stuttgart: Klett-Cotta.

Cross, K.F., \& Lynch, R.L. (1988). The SMART way to sustain and define success. National Productivity Review, 8(1), 23-33.

Dabholkar, P. A., Thorpe, D. I., \& Rentz, J. O. (1996). A measure of service quality for retail stores: scale development and validation. Journal of the Academy of Marketing Science, 24(1), 3-16.

Dainton, N. (2018). Feedback in der Hochschule. UTB-Band, Bd. 4891. Bern: Haupt.

Deming, W.E. (2000). The new economics. For industry, government, education (2. Aufl.). (S. 247). Camebridge: MIT Press.

DIN Deutsches Institut für Normung e. V. (2015). DIN EN ISO 9000 Qualitätsmanagementsysteme - Grundlagen und Begriffe (ISO 9000:2015).

DIN Deutsches Institut für Normung e. V. (2015). DIN EN ISO 9001 Qualitätsmanagementsysteme - Anforderungen (ISO 9001:2015).

DIN Deutsches Institut für Normung e. V. (2018). DIN EN ISO 9004 Qualitätsmanagement - Qualität einer Organisation - Anleitung zum Erreichen nachhaltigen Erfolgs (ISO 9004:2018).

Ebner, K. (2014). Ohne Klient kein Coaching. Der Einfluss von Klienteneigenschaften auf die Wirkung von Coaching. Dissertation. Berlin: wvb.

EFQM (2013). Das EFQM-Modell für excellence. European Foundation for Quality Management. Brüssel: EFQM.

Erpenbeck, J., \& von Rosenstiel, L. (2003). Einführung. In J. Erpenbeck \& L. von Rosenstiel (Hrsg.), Handbuch Kompetenzmessung (S. IX-XL). Stuttgart: Schäffer-Poeschel.

Fröchling, A. (2003). Schreibcoaching. Ein innovatives Beratungskonzept. Aachen: Shaker.

Gordon, W. J.J. (1961). Synectics: the development of creative capacity. New York: Harper.

Grieshammer, E., Liebetanz, F., Peters, N., \& Zegenhagen, J. (2013). Zukunftsmodell Schreibberatung. Eine Anleitung zur Begleitung von Schreibenden im Studium. Bd. 2. Baltmannsweiler: Schneider Verlag Hohengehren.

Hanke, K., \& Boehnke, K. (2018). Werte und ihre Bedeutung im Coaching. In S. Greif, H. Möller \& W. Scholl (Hrsg.), Handbuch Schlüsselkonzepte im Coaching (S. 657-665). Berlin: Springer Nature. https://doi.org/10.1007/978-3-662-49483-7.

Heller, J., \& Gallenmüller, K. (2016). Thesen über Königswege und Irrwege im Coaching. In C. Triebel \& al (Hrsg.), Qualität im Coaching. Denkanstöße und neue Ansätze: Wie Coaching mehr 
Wirkung und Klientenzufriedenheit bringt (S. 1-8). Berlin, Heidelberg: Springer. https://doi.org/10.1007/978-3-662-49058-7_1.

Heß, T., \& Roth, W.L. (2001). Professionelles Coaching. Eine Expertenbefragung zur Qualitätseinschätzung und -entwicklung. Heidelberg: Asanger.

Ianiro, P. M., \& Kauffeld, S. (2018). Annäherung an den CoachingProzess. Eine interaktionsanalytische Betrachtung. In S. Greif, H. Möller \& W. Scholl (Hrsg.), Handbuch Schlüsselkonzepte im Coaching (S. 41-52). Berlin: Springer. https://doi.org/10.1007/ 978-3-662-49483-7.

Ingaldi, M. (2018). Overview of the main methods of service quality analysis. Production Engineering Archives, 18, 54-59. https://doi. org/10.30657/pea.2018.18.10.

Kaplan, R. S., \& Norton, D. P. (2004). Strategy Maps. Der Weg immateriellen Werten zum materiellen Erfolg. Stuttgart: Schäffer-Poeschel. Aus dem Amerikanischen von Péter Horváth und Bernd Gaiser

Kirkpatrick, D.L. (1998). Evaluating training programs: the four levels. San Francisco: Berrett-Koehler Publishers.

Klemm, M. (2011). Schreibberatung und Schreibtraining. In K. Knapp, et al. (Hrsg.), Angewandte Linguistik: Ein Lehrbuch (3. Aufl. S. 120-142). Tübingen, Basel: Francke.

Klonek, F.E., Günter, A. V., \& Kauffeld, S. (2016). Damit Sie auch im Coaching bekommen, was auf der Verpackung steht: Qualitätssicherung von Coachings am Beispiel der Prozessanalyse im Motivational Interviewing. In C. Triebel \& al (Hrsg.), Qualität im Coaching. Denkanstöße und neue Ansätze: Wie Coaching mehr Wirkung und Klientenzufriedenheit bringt (S. 155-168). Berlin, Heidelberg: Springer. https://doi.org/10.1007/978-3-662-490587_1.

Klute-Wenig, S., \& Refflinghaus, R. (2020). Quality management for microenterprises and start-ups: is the ISO 9001 suitable? International Journal of Quality and Service Sciences, 12(1), 44-55. https://doi.org/10.1108/IJQSS-01-2018-0006.

König, E., \& Volmer, G. (2002). Systemisches Coaching. Handbuch für Führungskräfte, Berater und Trainer. Weinheim, Basel: Beltz.

König, E., \& Volmer, G. (2012). Handbuch Systemisches Coaching (2. Aufl.). Weinheim, Basel: Beltz.

Koubek, A. (Hrsg.). (2015). Praxisbuch ISO 9001:2015. Die neuen Anforderungen verstehen und umsetzen. München: Carl Hanser.

Kromrey, H. (2001). Evaluation - ein vielschichtiges Konzept. Begriff und Methodik von Evaluierung und Evaluationsforschung. Empfehlungen für die Praxis. Sozialwissenschaften und Berufspraxis, 24(2), 105-131.

Kuchen, C., \& Pedrun, P. (2006). Welche Kompetenzen braucht ein Coach? Das ideale Kompetenzprofil aus Sicht von Coachs (2. Studienarbeit). Zürich: Hochschule für Angewandte Psychologie.

Kuckartz, U. (2012). Qualitative Inhaltsanalyse. Methoden, Praxis, Computerunterstützung. Weinheim, Basel: Beltz Juventa.

Kühl, S. (2008). Coaching und Supervision. Personenorientierte Beratung in Organisationen. Wiesbaden: VS. https://doi.org/10.1007/ 978-3-531-91136-6.

Ladhari, R. (2008). Alternative measures of service quality: a review. Managing Service Quality: An International Journal, 18(1), 65-86.

Ladhari, R. (2009). A review of twenty years of SERVQUAL research. International Journal of Quality and Service Sciences, 1(2), 172-198. https://doi.org/10.1108/17566690910971445.

Leedham, M. (2005). The Coaching Scorecard: a holistic approach to evaluating the benefits of business coaching. International Journal of Evidence Based Coaching and Mentoring, 3(2), 30-44.

Lippmann, E. (2015). Was macht einen Coach zum Coach? Weiterbildung und Qualifizierung. Coaching Theorie \& Praxis, 1, 51-60. https://doi.org/10.1365/s40896-015-0008-3.

Martínez García, J. A., \& Martínez Caro, L. (2010a). Rethinking perceived service quality: An alternative to hierarchical and multidimensional models. Total Quality Management, 21(1), 93-118. https://doi.org/10.1080/14783360903492694.
Martínez García, J. A., \& Martínez Caro, L. (2010b). Some insights on conceptualizing and measuring service quality. Journal of Retailing and Consumer Services, 17(1), 29-42. https://doi.org/10. 1016/j.jretconser.2009.09.002.

Meyhöfer, F. (2016). Schreibberatung als Profession? Ein handlungslogischer Verortungsversuch. Journal der Schreibberatung, 11, 25-30. https://doi.org/10.3278/JOS1601W.

Möller, H., \& Kotte, S. (2018). Diagnostik im Coaching. In S. Greif, H. Möller \& W. Scholl (Hrsg.), Handbuch Schlüsselkonzepte im Coaching (S. 105-113). Berlin: Springer. https://doi.org/10.1007/ 978-3-662-49483-7.

Parasuraman, A., Zeithaml, V. A., \& Berry, L. L. (1985). A conceptual model of service quality and its implications for future research. Journal of Marketing, 49(4), 41-50.

Parasuraman, A., Zeithaml, V. A., \& Berry, L. L. (1988). SERVQUAL: a multiple-item scale for measuring consumer perceptions of service quality. Journal of Retailing, 64(1), 12-40.

Parasuraman, A., Zeithaml, V. A., \& Berry, L.L. (1991). Refinement and reassessment of the SERVQUAL scale. Journal of Retailing, 67(4), 420-450.

Parasuraman, A., Zeithaml, V. A., \& Berry, L.L. (1994). Alternative scales for measuring service quality: a comparative assessment based on psychometric and diagnostic criteria. Journal of Retailing, 70(3), 201-230.

Peters, N., \& Girgensohn, K. (2012). Studentische Schreibberatung ist professionell und persönlich. Ergebnisse einer Studie zu Peer Tutoring im Schreibzentrum. Zeitschrift Schreiben 12 (1), 1-9. https://www.researchgate.net/publication/263851376_ Studentische_Schreibberatung_ist_professionell_und_personlich _-_Ergebnisse_einer_Studie_zu_Peer_Tutoring_im_Schreibzen trum. Zugegriffen: 4. Aug. 2020.

Rascher, R. (2016). Reflexion und Management von Fehlern im Coaching. In C. Triebel \& al (Hrsg.), Qualität im Coaching. Denkanstöße und neue Ansätze: Wie Coaching mehr Wirkung und Klientenzufriedenheit bringt (S. 93-105). Berlin, Heidelberg: Springer. https://doi.org/10.1007/978-3-662-49058-7_1.

Rauen, C. (2014). Coaching (3. Aufl.). Göttingen: Hogrefe.

Reimer, A., \& Kuehn, R. (2005). The impact of servicescape on quality perception. European Journal of Marketing, 39(7/8), 785-808. https://doi.org/10.1108/03090560510601761.

Rojon, C., Bode, N., \& McDowall, A. (2020). What clients want: a conjoint analysis of precursors to coach selection. International Journal of Evidence Based Coaching and Mentoring, 18(1), 73-87. https://doi.org/10.24384/68qj-0r87.

Rust, R. T., \& Oliver, R.L. (1994). Service quality: insights and managerial implications from the frontier. In R. T. Rust \& R. L. Oliver (Hrsg.), Service quality: new directions in theory and practice (S. 1-19). London: SAGE.

Schermuly, C.C., \& Graßmann, C. (2015). Erfolgreicher Einsatz von Coaching in der Führungskräfteentwicklung. In J. Felfe \& R. van Dick (Hrsg.), Handbuch Mitarbeiterführung: Wirtschaftspsychologisches Praxiswissen für Fach- und Führungskräfte (S. 1-13). Heidelberg: Springer. https://doi.org/10.1007/978-3-642-550805.

Schmidt-Lellek, C. J. (2006). Anmerkungen zur Professionalisierung des Coaching auf dem Hintergrund des klassischen Professionsbegriffs. Organisationsberatung, Supervision, Coaching, 13, 183-192. https://doi.org/10.1007/s11613-006-0025-1.

Schmidtmayer, A. (2016). Steuerung der Prozessqualität im Coaching mit der Ampelsystematik. In C. Triebel \& al (Hrsg.), Qualität im Coaching. Denkanstöße und neue Ansätze: Wie Coaching mehr Wirkung und Klientenzufriedenheit bringt (S. 169-181). Berlin, Heidelberg: Springer. https://doi.org/10.1007/978-3-662-490587 1.

Schneider, B., \& White, S. S. (2004). Service quality: research perspectives (Foundations for Organizational Science). Thousand Oaks, London, New Delhi: SAGE.

Schreyögg, A. (2000). Editorial. Organisationsberatung Supervision Coaching, 7(1), 3-8. https://doi.org/10.1007/s11613-000-0002-z. 
Seidl, T. (2013). Konzeptionelle Überlegungen zum ,Schreibenden Coaching' an der Hochschule. Journal der Schreibberatung, 6, 9-22. https://doi.org/10.3278/JOS1301W.

Steinke, I. (2010). Modelle des Qualitätsmanagements im Coaching. Organisationsberatung Supervision Coaching, 17(4), 415-438. https://doi.org/10.1007/s11613-010-0212-y.

Triebel, C., \& Heller, J. (2016). Vorwort. In C. Triebel \& al (Hrsg.), Qualität im Coaching. Denkanstöße und neue Ansätze: Wie Coaching mehr Wirkung und Klientenzufriedenheit bringt (S. V-VII). Berlin, Heidelberg: Springer. https://doi.org/10.1007/978-3-66249058-7_1.

Ulmi, M., Bürki, G., Verein, A., \& Marti, M. (2017). Textdiagnose und Schreibberatung (2. Aufl.). Opladen, Berlin, Toronto: Barbara Budrich.

Varnali, K. (2019). Understanding customer journey from the lenses of complexity theory. The Service Industries Journal, 39(11-12), 820-835. https://doi.org/10.1080/02642069.2018.1445725.

Wagner, K. W., \& Patzak, G. (2015). Performance Excellence. Der Praxisleitfaden zum effektiven Prozessmanagement (2. Aufl.). München: Hanser.
Wastian, M., \& Poetschki, J. (2016). Zielklärung und Zielerreichung im Coaching. Coaching Theorie \& Praxis, 2, 21-31. https://doi. org/10.1365/s40896-016-0011-3.

Webers, T. (2016). Kompetenzen zur Steuerung des Coaching-Prozesses. In C. Triebel \& al (Hrsg.), Qualität im Coaching. Denkanstöße und neue Ansätze: Wie Coaching mehr Wirkung und Klientenzufriedenheit bringt (S. 71-79). Berlin, Heidelberg: Springer. https://doi.org/10.1007/978-3-662-49058-7_1.

Webers, T., \& Zickermann, R. (2019). Training vs. Coaching - über Aporien in der Professionalisierungsdiskussion. Coaching Theorie \& Praxis, 5, 35-50. https://doi.org/10.1365/s40896-01900030-z.

von Werder, L., Schulte-Steinicke, B., \& Schulte, B. (2001). Weg mit Schreibstörung und Lesestress. Zur Praxis und Psychologie des Schreib- und Lesecoachings. Baltmannsweiler: Schneider-Verlag Hohengehren.

Publisher's Note Springer Nature remains neutral with regard to jurisdictional claims in published maps and institutional affiliations. 